\title{
Acute lung injury \& acute respiratory distress syndrome - part I
}

\section{Summary}

NSAIDs are commonly used as single analgesics in minor surgery or as component of multimodal analgesia associated with opioids or locoregional techniques in the postoperative period to assure a better analgesia and reduce the dose of opioids. The analgesic potency evaluated as number needed to treat (NNT) is not very different between the traditional non selective NSAIDs and the selective cyclo-oxygenase-2-inhibitors (Coxibs). The effectiveness as analgesics is unquestionable also if these drugs are not devoid of risks. There is debate in literature about the possible side effects when administered in the perioperative period: anastomotic leakage, reduced ossification, bleeding and acute renal failure. Recent data underline as the Coxibs but also traditional NSAIDs can induce cardiac toxicity even if they are utilized for few days. The aim of this review is to provide an overview of the effectiveness and side effects of selective and non-selective NSAIDs in the perioperative period.
Volume 3 Issue 5 - 2015

\section{Fadhil Zwer}

Consultant Intensive Care Medicine at Private practice clinic, Iraq

Correspondence: Fadhil Zwer, Consultant Intensive Care Medicine at Private practice clinic, Iraq,

Email drfadhil2@gmail.com

Received: August 28, 2015 | Published: November 26, 2015

\section{History}

There are many interesting historical medical events that correlated with or impacted the identification and management of ARDS leading to the most new modern current concepts. These important and dramatic events set the stage for the critical care revolution that occurred in the last half of the twentieth century (Table 1). ${ }^{1}$

In 1821 , in what was probably the first published scientific description, Laennec described the gross pathology of the heart and lungs and described the idiopathic changes of the lungs; pulmonary Edema without heart failure in [A Treatise on Diseases of the Chest]. By the 1950s, pulmonary edema had

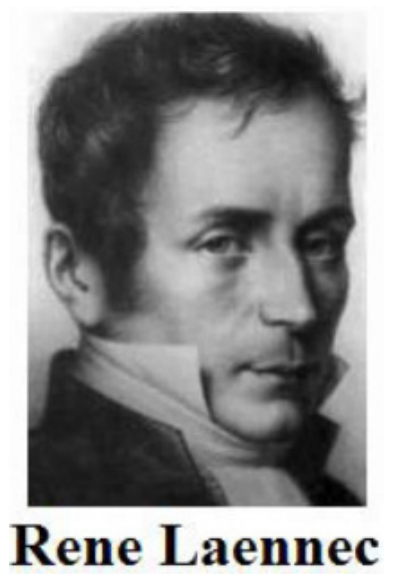

become a medical subject heading by the National Library of Medicine; however, no distinction was made at that time between cardiac and non cardiac causes. Nonetheless, what evidently moved ARDS from a nearly universally fatal form of double pneumonia was the development of methods of establishing secure airway access using tubes that could be attached to mechanical ventilators to deliver adequate pulmonary distending pressures. These techniques extended the lives of patients from a few hours to many days or even weeks, that is a long enough to recover in some cases. As this new kind of patient began to populate the newly established intensive care units, their condition rapidly became recognized as one of the most challenging acute clinical processes to treat. Since acute, diffuse, and dense bilateral infiltrates were almost never observed except in patients requiring prolonged mechanical ventilation, many surmised the cause of such infiltrates was the ventilator, hence the term [respirator lung].

For a period of time ARDS had taken the name of inciting injuries (e.g., DaNang lung, shock lung, post-traumatic lung, etc.). It wasn't until 1967, in a landmark article published in Lancet, that Ashbaugh, Bigelow, Petty, and Levine ${ }^{2}$ first described the clinical entity that they called [acute respiratory distress in adults]. This article recognized for the first time that ARDS was a disarrangement of pathophysiologic abnormalities common to 10 a relatively large number of patients but that were initiated by a wide variety of unrelated insults for example, gastric aspiration, sepsis, blunt trauma, near-drowning, etc. Interestingly, the difficulty in making the diagnosis remained evident in that at least five of the patients studied could have had ARDS secondary to or complicated by fluid overload. Also notable in this 1967 report, ARDS was acute respiratory distress syndrome. However, in 1971 Petty and Ashbaugh used the term adult respiratory distress syndrome in another publication probably to address the perception of ARDS as an adult version of the previously described infant respiratory distress syndrome (IRDS). The ARDS term firstly used as an adult respiratory distress syndrome but with more advances in researches and with more clarity of the syndrome which make diagnosis of the syndrome more accurate, it was found that this disorder may attacks not only the adult patients but even the children, so the term was changed from adult to acute respiratory distress syndrome.

In 1992 the American European Consensus Conference (AECC) was charged with developing a standardized definition for ARDS to assist with clinical and epidemiologic research. The AECC recommended that a new designation, acute lung injury (ALI), be defined as a syndrome of inflammation and increased permeability that is associated with a changes of clinical, radiologic, and physiologic abnormalities that cannot be explained by, but may coexist with, left atrial or pulmonary capillary hypertension and is associated most often with sepsis syndrome, aspiration, primary pneumonia, or multiple trauma and less commonly with cardiopulmonary bypass, multiple transfusions, fat embolism, pancreatitis, and others. Acute (not 
adult) respiratory distress syndrome was defined as a subset of ALI patients with more severe oxygenation defect (more hypoxemia). ALI and ARDS are acute in onset and persistent, associated with one or more known risk factors, and are characterized by arterial hypoxemia resistant to oxygen therapy alone and diffuse radiologic infiltrates. In the decade of the 1970s ARDS became increasingly recognized, but hydrostatic causes (e.g., volume overload) were difficult to rule out. The potential for confusion was so great that measurements of pulmonary artery wedge pressure became a very common means of diagnosis. In fact, entry criteria for one of the earliest randomized trials of corticosteroids required a measured pulmonary wedge pressure less than $19 \mathrm{mmHg}$. This once nearly routine measurement is now much less frequent with the realization that exceeding an arbitrary pulmonary artery occlusion pressures (PAOP) does not exclude the diagnosis of ALI, and that there are usually other clinical data and historical clues that allow a fairly secure diagnosis of ALI apart from volume overload to be made. Even when the PAOP is less than 18 $\mathrm{mm} \mathrm{Hg}$, one cannot be certain that edema is the result of altered permeability.

Table I Medical events that made modern critical care possible

\begin{tabular}{|c|c|}
\hline Year & Event \\
\hline 1896 & Roentgen describes X-rays. Early chest X-rays required exposure time of $20 \mathrm{~min}$ and were not considered useful until the 1920 s. \\
\hline 1929 & Drinker and Shaw announce the invention of the iron lung during the polio epidemic. \\
\hline 1946 & $\begin{array}{l}\text { Fenn and coworkers publish work on pulmonary gas exchange, relating PO2, PCO2, respiratory exchange ratio, arterial O2 } \\
\text { saturation, alveolar ventilation, and altitude. }\end{array}$ \\
\hline 1950 & Cortisone, first extracted in 1936 , is used as a treatment for asthma. \\
\hline 1954 & Clark develops an electrode for measurement of $\mathrm{PO} 2$, reflecting the level of $\mathrm{O} 2$ in the blood. \\
\hline 1954 & Ibsen first used cuffed entdotracheal tubes and positive pressure ventilation. \\
\hline 1956 & $\begin{array}{l}\text { Forssmann and colleagues share the Nobel Prize in physiology and medicine for the advancement of right-heart catherization. Their } \\
\text { work led to the development of the Swan Ganz catheter. }\end{array}$ \\
\hline 1959 & $\begin{array}{l}\text { Avery and Mead discover that some newborns' lungs do not produce surfactant (hyaline membrane disease, later called neonatal } \\
\text { respiratory distress syndrome). }\end{array}$ \\
\hline 1960 & Moss and coworkers discover the benefits of chest compression to achieve artificial circulation. \\
\hline 1967 & Ashbaugh and colleagues identify acute respiratory distress syndrome. \\
\hline $1960 \mathrm{~s}$ & First developed in the 1950s, it was the late 1960s before intensive care units become common in the United States. \\
\hline 1976 & $\begin{array}{l}\text { Extracorporeal membrane oxygenation (ECMO) is introduced for neonates with persistent pulmonary hypertension, allowing the } \\
\text { lungs time to heal. }\end{array}$ \\
\hline 1978 & $\begin{array}{l}\text { Furchgott describes "endothelium-derived relaxing factor," now known as nitric oxide. He was awarded the Nobel Prize for this } \\
\text { work. }\end{array}$ \\
\hline
\end{tabular}

Table 2 Definitions of the Acute Respiratory Distress Syndrome

\begin{tabular}{|c|c|c|c|c|}
\hline Study & No of Patients & Patients PaO2/FiO2 Ratio & Mortality & \\
\hline \multirow{6}{*}{ Meade 2008} & \multirow{6}{*}{983} & & Hospital mortality & \\
\hline & & & Lung open vent. & Control \\
\hline & & $41-106$ & 50 & 58 \\
\hline & & $>106-142$ & 39 & 43 \\
\hline & & $>142-180$ & 33 & 33 \\
\hline & & $>180-250$ & 25 & 26 \\
\hline \multirow{4}{*}{ Villar 20II } & \multirow{4}{*}{220} & & Hospital mortality & \\
\hline & & $<112$ & 47 & \\
\hline & & $112-142$ & 30 & \\
\hline & & $>142$ & 23 & \\
\hline \multirow{4}{*}{ Cooke 2008} & \multirow{4}{*}{1113} & & Hospital mortality & \\
\hline & & $\leq 100$ & 50 & \\
\hline & & $\leq 100+$ shock & 58 & \\
\hline & & $\leq 100+$ oliguric renal failure & 71 & \\
\hline \multirow{4}{*}{ Villar 2007} & \multirow{4}{*}{170} & & ICU mortality & Hospital mortality \\
\hline & & $<112$ & 45 & 45 \\
\hline & & $112-142$ & 20 & 20 \\
\hline & & $>142$ & 6.3 & 12.6 \\
\hline & \multirow{4}{*}{1113} & & Hospital mortality & \\
\hline \multirow{4}{*}{ Rubenfeld 2005} & & $<200$ (ARDS) & 41.1 & \\
\hline & & $200-3000$ (ALI) & 38.5 & \\
\hline & & ALI progressed to ARDS on day 3 or day 7 & $4 I$ & \\
\hline & & ALI no progression to ARDS on day 3 or day 7 & 28.6 & \\
\hline \multirow{3}{*}{ Brun-Buisson ALIVE study 2004} & \multirow{3}{*}{463} & & ICU mortality & Hospital mortality \\
\hline & & $<200$ (ARDS) & 49.4 & 57.9 \\
\hline & & $200-300(\mathrm{ALI})$ & 22.6 & 32.7 \\
\hline & & & ICU mortality & \\
\hline \multirow{5}{*}{ Esteban 2002} & \multirow{4}{*}{120} & $<100$ & 83 & \\
\hline & & $100-149$ & 47 & \\
\hline & & $150-199$ & 31 & \\
\hline & & $200-300$ & 25 & \\
\hline & \multirow{4}{*}{342} & $>300$ & 24 & \\
\hline \multirow{3}{*}{ Taccone Prone-Supine II 2009} & & & ICU mortality & Hospital mortality \\
\hline & & $<100$ (sever ARDS) & 42 & 50.7 \\
\hline & & $100-200$ (ARDS) & 24 & 31.8 \\
\hline
\end{tabular}


Table 3 Severity of hypoxemia and outcome in ALI/ARDS 3

Part I Acute or chronic, depending on course.

Part 2 Severity of physiological lung injury as determined by the lung injury score (see table I-5).

Part 3 Lung injury caused by or associated with known risk factor for ARDS such as sepsis, pneumonia, aspiration, or major trauma.

Table 4 Three part expanded definition of clinical acute lung injury (ALI) and the acute respiratory distress syndrome (ARDS) proposed by Murray and colleagues $^{6}$

\begin{tabular}{|c|c|c|}
\hline \multirow[t]{3}{*}{ Clinical Data } & & Score \\
\hline & No alveolar consolidation & 0 \\
\hline & Alveolar consolidation confined to I quadrant & 1 \\
\hline \multirow[t]{5}{*}{ Chest radiography } & Alveolar consolidation confined to 2 quadrants & 2 \\
\hline & Alveolar consolidation confined to 3 quadrants & 3 \\
\hline & Alveolar consolidation confined to 4 quadrants & 4 \\
\hline & $\mathrm{PaO}_{2} / \mathrm{FiO}_{2} \geq 3000$ & 0 \\
\hline & $\mathrm{PaO}_{2} / \mathrm{FiO}_{2} 225-299$ & I \\
\hline \multirow[t]{5}{*}{ Hypoxemia score } & $\mathrm{PaO}_{2} / \mathrm{FiO}_{2}, \mathrm{I75-224}$ & 2 \\
\hline & $\mathrm{PaO}_{2} / \mathrm{FiO}_{2} \mathrm{I00}-\mathrm{I} 74$ & 3 \\
\hline & $\mathrm{PaO}_{2} / \mathrm{FiO}_{2}<100$ & 4 \\
\hline & $\leq 5 \mathrm{~cm} \mathrm{H} \mathrm{H}_{2} \mathrm{O}$ & 0 \\
\hline & $6-8 \mathrm{~cm} \mathrm{H} \mathrm{H}_{2} \mathrm{O}$ & 1 \\
\hline \multirow[t]{5}{*}{ PEEP score (when mechanically vent.) } & $9-1 \mathrm{lcm} \mathrm{H} \mathrm{C}_{2} \mathrm{O}$ & 2 \\
\hline & $12-14 \mathrm{~cm} \mathrm{H}_{2} \mathrm{O}$ & 3 \\
\hline & $\geq 15 \mathrm{~cm} \mathrm{H}_{2} \mathrm{O}$ & 4 \\
\hline & $\geq 80 \mathrm{ml} / \mathrm{cm} \mathrm{H}_{2} \mathrm{O}$ & 0 \\
\hline & $60-79 \mathrm{ml} / \mathrm{cm} \mathrm{H} \mathrm{H}_{2} \mathrm{O}$ & 1 \\
\hline \multirow[t]{3}{*}{ Respiratory system compliance score (when available) } & $40-59 \mathrm{ml} / \mathrm{cm} \mathrm{H} \mathrm{H}_{2} \mathrm{O}$ & 2 \\
\hline & $20-39 \mathrm{ml} / \mathrm{cm} \mathrm{H} \mathrm{H}_{2} \mathrm{O}$ & 3 \\
\hline & $\leq 19 \mathrm{ml} / \mathrm{cm} \mathrm{H} \mathrm{H}_{2} \mathrm{O}$ & 4 \\
\hline
\end{tabular}

Table 5 Calculation of the lung injury score ${ }^{6}$

\begin{tabular}{|c|c|c|c|}
\hline Criteria & Timing & Oxygenation & Pulmonary artery wedge pressure \\
\hline ALI criteria & Acute onset & $\begin{array}{l}\mathrm{PaO}_{2} / \mathrm{FiO}_{2} \leq 300 \mathrm{mmHg} \\
\text { (regardless of PEEP level) }\end{array}$ & $\begin{array}{l}\text { Bilateral infiltrates seen on frontal chest } \leq 18 \mathrm{mmHg} \text { when measured or no clinical } \\
\text { radiograph } \\
\text { evidence of left atrial hypertension }\end{array}$ \\
\hline ARDS criteria & Acute onset & $\begin{array}{l}\mathrm{PaO}_{2} / \mathrm{FiO}_{2} \leq 200 \mathrm{mmHg} \\
\text { (regardless of PEEP level) }\end{array}$ & $\begin{array}{l}\text { Bilateral infiltrates seen on frontal chest } \leq 18 \mathrm{mmHg} \text { when measured or no clinical } \\
\text { radiograph chest radiograph }\end{array}$ \\
\hline
\end{tabular}

The score is calculated by adding the sum of each component and dividing by the number of components used

No lung injury -0

Mild to moderate lung injury - 0.1-2.5

Sever lung injury (ARDS) - >2.5

Table 6 Recommended Criteria for Acute Lung Injury and Acute Respiratory Distress Syndrome ${ }^{21}$

\begin{tabular}{|c|c|c|c|c|}
\hline PEEP $\leq 5$ & $\mathbf{5}<$ PEEP $\leq \mathbf{I 0}$ & $\mathbf{I} \mathbf{I} \geq \mathbf{P E P}$ & Total** & \\
\hline $\mathrm{PaO}_{2} / \mathrm{FiO}_{2}>175$ & $23 . I \pm 5 \%$ & $22.0 \pm 6 \%$ & $25.9 \pm 18 \%$ & $23.1 \pm 2 \%$ \\
\hline $\mathrm{II} 0<\mathrm{PaO}_{2} / \mathrm{FiO}_{2} \leq 175$ & $31.4 \pm 9 \%$ & $25.4 \pm 5 \%$ & $28.1 \pm 13 \%$ & $27.8 \pm 3 \%$ \\
\hline $\mathrm{PaO}_{2} / \mathrm{FiO}_{2} \leq 115$ & $35.7 \pm 2 \%$ & $35.2 \pm 7 \%$ & $38.2 \pm 7 \%$ & $36.5 \pm 3 \%$ \\
\hline Total $*$ & $27.8 \pm 3 \%$ & $27.8 \pm 2 \%$ & $33.3 \pm 4 \%$ & \\
\hline
\end{tabular}

Table 7 Mortality rates according to $\mathrm{PaO}_{2} / \mathrm{FiO}_{2}$ tertiles and PEEP levels ${ }^{6}$

\begin{tabular}{ll}
\hline Defining Characteristic & Operational Definition \\
\hline I Hypoxemia & $\mathrm{PaO}_{2} / \mathrm{FiO}_{2}<200 \mathrm{mmHg}$ with PEEP $\geq \mathrm{I0}$. \\
2 Acute onset & Rapid onset in $<72$ hours. \\
3 Radiographic abnormalities & Bilateral airspace disease involving $\geq 2$ quadrants on frontal chest $x$ ray. \\
4 Non cardiogenic in origin & No clinical evidence of congestive heart failure (including use of pulmonary artery catheter and/or echo if clinically indicated). \\
5 Decreased lung compliance & Static respiratory system compliance $<50 \mathrm{ml} / \mathrm{cm} \mathrm{H}_{2} \mathrm{O}$ (with patient sedated, tidal volume of $8 \mathrm{ml} / \mathrm{kg}$, ideal body weight, $\mathrm{PEEP} \geq$ \\
6 Predisposition & $\mathrm{I}$ ). \\
\hline
\end{tabular}

-Overall mortality was $29.2 \% \pm$ values are standard errors. Data analyzed using Cochran-Armitage trend test.

*Within each PEEP range there is a highly significant increase in mortality with lower $\mathrm{PaO}_{2} / \mathrm{FiO}_{2}$ levels $(\mathrm{P}<0.000 \mathrm{I})$.

** Within each $\mathrm{PaO}_{2} / \mathrm{FiO}_{2}$ tertile there is no significant difference in mortality with increasing PEEP level. 
Table 8 The Delphi definition of ARDS ${ }^{21}$

\section{Acute Respiratory Distress Syndrome}

Timing

Chest imaging*

Origin of Edema

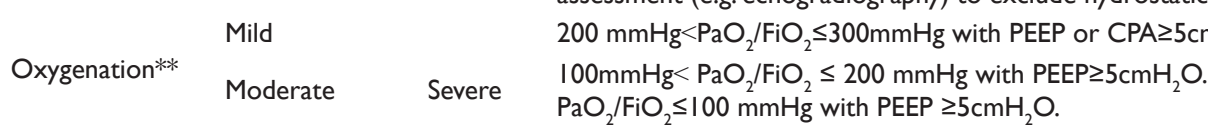

Within I week of a known clinical insult or new worsening respiratory symptoms.

Bilateral opacities - not fully explained by effusion, lobar/lung collapse or nodules.

Respiratory failure not fully explained by cardiac failure or fluid overload need objective assessment (e.g. echogradiography) to exclude hydrostatic Edema if no risk factor present.

ARDS is indicated by the presence I of criteria -4 and one of 5 or 6 .

Table 9 The Berlin definition of ARDS (the ARDS definition task force: the Draft Berlin Definition, ESICM 24 the Annual Congress Berlin, October 20I I)

\begin{tabular}{lll}
\hline ARDS severity & PaO2/FiO2 * & Mortality ** \\
\hline Mild & $200-300$ & $27 \%$ \\
Moderate & $100-200$ & $32 \%$ \\
Sever & $<100$ & $45 \%$ \\
\hline
\end{tabular}

$*$ = chest radiograph or CTscan.

$* *=$ if altitude is higher than $1000 \mathrm{~m}$, the correction factor should be calculated as follow: $\left[\mathrm{PaO}_{2} / \mathrm{FiO}{ }_{2} \times(\right.$ barometric pressure/760) $]$.

$* * *=$ this may be delivered non-invasively in the mild acute respiratory distress syndrome group.

Table I 0 Berline definition of ARDS and mortality rate r2 $^{22}$

\begin{tabular}{ll}
\hline Pulmonary ARDS & Extrapulmonary ARDS \\
\hline Bacterial, fungal, viral and parasitic pneumonia. & Sepsis. \\
Aspiration of gastric content. & Trauma. \\
Pulmonary contusion. & Drug overdose. \\
Inhalation injury. & Acute pancreatitis. \\
Fat emboli. & Cardiopulmonary bypass. \\
\hline
\end{tabular}

$*=$ on PEEP $5+* *=$ observed in cohort.

Table I I Underlying etiologies of pulmonary and extrapulmonary acute respiratory distress syndrome ${ }^{19}$

\begin{tabular}{llll}
\hline Pulmonary ARDS & Extrapulmonary ARDS & & \\
\cline { 1 - 2 } & Alveolar epithelium & $\uparrow \uparrow$ damage & Damage \\
& Altered type I and II cell & $\uparrow \uparrow$ damage & Normal \\
& Alveolar neutrophils & Prevalent & Rare \\
& Apoptotic neutrophils & Prevalent & Rare \\
& Fibrinous exudate & Present & Rare \\
& Alveolar collapse & $\uparrow \uparrow$ increased & Increased \\
& Local interleukin & Prevalent & Rare \\
& Interstitial edema & Absent & High \\
Interstitial space & Collagen fibers & $\uparrow \uparrow$ increased & Increased \\
& Elastic fibers & Normal & Normal \\
& Capillary endothelium & Normal & $\uparrow \uparrow$ damage \\
Blood & Interleukin & Increased & $\uparrow \uparrow$ increased \\
& TNF- $\alpha$ & Increased & $\uparrow \uparrow$ increased \\
\hline
\end{tabular}

Table 12 Histological and biochemical alterations in pulmonary and extrapulmonary acute respiratory distress syndrome ${ }^{19}$

\begin{tabular}{|c|c|c|c|c|c|c|}
\hline \multicolumn{2}{|l|}{ Exposure Frequency } & \multicolumn{2}{|c|}{ Discordant pairs* } & \multicolumn{3}{|c|}{ Concordant pairs* } \\
\hline Variables & Recurrent ALI\% & Single ALI\% & $+1-$ & ++ & $+/+$ & + \\
\hline Gastro-esophageal reflex disease & 79 & 26 & 11 & I & 4 & 3 \\
\hline Proton pump inhibitors use & 42 & 16 & 6 & I & 2 & 10 \\
\hline $\mathrm{H} 2$ blocker use & 32 & II & 5 & I & I & 12 \\
\hline Aspiration of gastric contents & 37 & 16 & 5 & I & 2 & II \\
\hline Chronic pain & 5 & 0 & I & 0 & 0 & 18 \\
\hline Chronic opioid use & 21 & 0 & 4 & 0 & 0 & 15 \\
\hline Smoking & 68 & 47 & 8 & 4 & 5 & 2 \\
\hline Chronic alcohol use & 10 & 10 & 2 & 2 & 0 & 15 \\
\hline Obstructive lung disease & 31.6 & 21.1 & 4 & 2 & 2 & II \\
\hline Immunocompromised & 10.5 & 5.3 & 2 & I & 0 & 16 \\
\hline Diabetes mellitus & 31.6 & 15.7 & 5 & 2 & I & II \\
\hline Body mass index $(\mathrm{BMI}) \geq 30$ & 31.6 & 4 & 5 & 3 & I & 10 \\
\hline Transfusion & 26 & 32 & 3 & 4 & 2 & 10 \\
\hline
\end{tabular}


Reduced colloid oncotic pressure as observed in hypo albuminemic states promotes edema in the absence of permeability changes. Lung dysfunction secondary to direct insults to the lung, for example, aspiration (primary ARDS), may be different from lung injury from extra pulmonary origin, for example, sepsis (secondary ARDS). This is an interesting concept, and some clinical markers that differentiate the two have been described. However, work is still underway to determine implications for patient management. The differential diagnosis of ARDS using the AECC definition, is significantly broad that the resulting list of other potential confusing diagnoses is limited to diseases that appear similar with regard to chest radiography and oxygenation defect, but are sub acute or chronic: interstitial or idiopathic pulmonary fibrosis, lymphangitic carcinoma, pulmonary veno occlusive disease; or are due to increased hydrostatic pressures: mitral stenosis, left ventricular failure, intravascular volume overload. Thus the diagnosis of ARDS is inclusive and indeed can coexist with other more specific diagnoses such as pneumococcal pneumonia.

As mentioned above, the first description of acute respiratory distress syndrome appeared in 1967, in a paper by Ashbaugh et al. ${ }^{2}$ which described 12 patients with acute respiratory distress, cyanosis refractory to oxygen therapy, decrease lung compliance, and diffuse infiltrates on chest radiography. In 1988, an expanded definition was proposed based on the level of positive end-expiratory pressure (PEEP), the ratio of the partial pressure of arterial oxygen to the fraction of inspired oxygen, the static lung compliance, and the degree of infiltration evident on chest radiography. Another measurement, the lung injury score, has been widely used to quantify the disease severity in clinical trials, although it has not been shown to accurately predict outcome during the first 24 to 72 hours after the onset of ARDS.

In 1994, a new definition was recommended by the AmericanEuropean Consensus Conference Committee (Table 2), which recognized the variability in severity of lung injury, and separated patients into two groups: those with less severe hypoxemia were categorized as having acute lung injury and those with more severe hypoxemia were defined as having ARDS. However, factors such as the underlying cause and involvement of other organ systems are not a part of the existing definition (Table 2).

Abbreviations: IRDS, infant respiratory distress syndrome; AECC, american european consensus conference; ALI: acute lung injury; PAOP: pulmonary artery occlusion pressures; PEEP, positive end-expiratory pressure; $\mathrm{ABG}$, arterial blood gas; LIS, lung injury score; AECC, american-european consensus conference; OI, oxygenation index; MAP, mean airway pressure; ATS, american thoracic society; SCCM, society of critical care medicine; TNF, tumor necrosis factor; BAL, broncho alveolar Lavage; CT, computed tomography; APACHE, acute physiology and chronic health evaluation; LIPS, lung injury prediction score

\section{Definitions of ALI/ARDS}

The acute lung injury and acute respiratory distress syndrome are characterized by increased permeability of the alveolarcapillary membrane, diffuse alveolar damage, the accumulation of proteinaceous interstitial and intra-alveolar edema, and the presence of hyaline membranes. These pathological changes are accompanied by physiological alterations including severe hypoxemia, an increase in the mean pulmonary dead-space fraction, and a decrease in pulmonary compliance. ARDS is a relatively common diagnosis in patients who require mechanical ventilation for greater than 24 hours. In a population based cohort study of 21 hospitals over a 16-month period of time, $21 \%$ of patients who required mechanical ventilation for more than 24 hours met established criteria for ARDS. A European survey of 132 intensive care units similarly demonstrated that $18 \%$ of mechanically ventilated patients mostly had ARDS. Because ALI/ARDS is a syndrome and not a disease, patients are defined as having ALI/ARDS when they meet predetermined diagnostic criteria. Potential goals for these diagnostic criteria are to identify patients with a specific clinical entity for epidemiological purposes and to select patients who will respond to ALI/ARDS specific therapies. The diagnostic criteria used to define ALI/ARDS have progressed over time.

For the purpose of clearing an essential one of criteria [which will mention frequently in the discussion] for definition of ALI/ARDS, so the abbreviation of $\mathrm{PaO}_{2} / \mathrm{FiO}_{2}$ must be clarified (see the box bellow). A $\mathrm{PaO}_{2} / \mathrm{FiO}_{2}$ ratio is an index to characterize the ALI/ARDS, which involves severe hypoxemia (insufficient oxygen content in blood). $\mathrm{PaO}_{2}$ is the partial pressure of oxygen in arterial blood. It's usually measured in millimeters of mercury ( $\mathrm{mmHg}$ or Torr) by the test called arterial blood gas (ABG) analysis. $\mathrm{PaO}_{2}$ of 75 to $100 \mathrm{mmHg}$ is considered normal. $\mathrm{FiO}_{2}$ is the fraction of inspired oxygen or, simply percentage of oxygen, in a gas mixture atmospheric or given by medical gas equipments. For example, the atmospheric air has $\mathrm{FiO}_{2}$ of $21 \%(0.21)$. If a patient needs mechanical ventilation, $\mathrm{FiO}_{2}$ is usually in the $30-$ to- $40 \%$ range. Therefore, the normal ratios in condition of patient inhaling spontaneously [atmospheric air] without incremental oxygen will be:

$\mathrm{PaO}_{2} / \mathrm{FiO}_{2}=75 / 0.21=357$ [lower acceptable ratio]

$85 / 0.21=404$

$95 / 0.21=452$

\section{$100 / 0.21=476$ [upper ratio]}

Although ALI/ARDS are syndromes caused by different injuries and conditions, the patho-biology of the lung injury and similar clinical picture makes an obligating state for us to study them as a single entity rather than characterize the individual risk factors as separate clinical entities. More importantly an array of potential specific targets for pharmacologic intervention can be applied to ALI/ARDS as a disease entity as a whole. The clinical presentations consistent with ALI and ARDS can arise in patients of all ages from direct (pulmonary) or indirect (extra pulmonary) insults that induce pulmonary inflammation, damage the cells of the alveolar-capillary membrane, and lead to severe acute respiratory failure. Uniform diagnostic criteria are essential for meaningful clinical studies and therapeutic development for ALI/ARDS. The clinical entities of ALI and ARDS are syndromes defined by a relatively limited set of descriptive pathophysiologic clinical findings, and patients are included regardless of the specific etiology of acute pulmonary dysfunction. Although a working definition of ALI/ARDS that includes both pulmonary and extra pulmonary causes can have benefit in standardizing supportive intensive care, it can also complicate assessments of the efficacy of therapeutic interventions. For example, the lack of stratification of patients with ALI/ARDS by etiologic causes has the potential to confound Data interpretation in therapeutic trials, since interventions that might benefit one cause of ALI/ARDS may have no benefit or may even be harmful in treating another etiology.

Anyhow, there exist major differences in the pathogenesis of these individual insults especially when studied in animal model systems. However, there are major advantages of defining a syndrome like ALI/ARDS: 
1. A standardized universal definition for ALI/ARDS has many benefits. Most importantly, it would allow comparison of the findings of various clinical trials in ALI/ARDS with a greater degree of certainty. For the clinician, a functional definition of ALI/ARDS allows early institution of standardized clinical care, i.e. certain therapeutic modalities that have been tested and proven to have benefits. For instance, early identification of patients with ALI/ARDS allows the early application of protective lung ventilation with lower tidal volumes based on predicted body weights.

2. Additionally, a standardized definition including ALI and ARDS can assist with outcome prognostication and is of help especially while discussing the care of the patient with families. For the researcher, it helps to capture a larger patient population for potential recruitment into large clinical studies, as proven by multiple clinical trials conducted under the observations of the ARDS network. Moreover, it offers a common language of communication between the basic and clinical researcher where therapeutic modalities can be constantly tested in the laboratory and brought to the clinical practice.

3. It has to be understood that while combining both ALI and ARDS as one entity offers advantages, the results of the clinical studies with testing of therapeutic modalities have to be carefully interpreted, as many specific discrete disease entities have been examined as one.

The traditional thinking with ARDS is that it is the multi-organ dysfunction and not hypoxemia that is responsible for mortality. This was based on a number of ARDS network trials where hypoxia was well tolerated (up to saturations of $88 \%$ ) and improvements in oxygenation did not lead to a survival advantage. It is important to point out that some studies after the adoption of lung protective strategies have suggested a strong correlation between the severity of hypoxemia and ICU or hospital mortality (Table 3$){ }^{3}$ In light of this, it is recommended that in future clinical trials of ALI/ARDS, severity of hypoxemia should be considered upon patient enrollment into the study, and that outcomes should be assessed based on severity of hypoxemia. What could be the reasons for these observations? One can speculate on the following possibilities. In patients with severe hypoxia, there is perhaps increased incidence of hyperoxia-induced lung injury from increased requirements of $\mathrm{FiO}_{2}$ to maintain saturations of $88 \%$. Hyperoxia has been implicated as a factor responsible for increased lung injury in many animal models by the generation of reactive oxygen species, increased apoptosis and necrosis. In addition it is important to consider the patho-physiological disturbances linking ARDS with multi-organ dysfunction. Is the multi-organ dysfunction as a result of ALI/ARDS or is the ALI/ARDS a result of multi-organ dysfunction? Are these two separate entities? These questions need to be fully explored in additional studies through basic and clinical research. Anyhow, over the past few years many different study groups have raised doubts about the validity of the current ALI/ARDS definitions and have recommended a change. It is strongly felling that it is time to change the definition after 17 years of the predominant use of the AECC definition for ALI/ARDS. Based on available data with various validity studies it is suggested that the new definition should be standardized as follows:

a. Risk factors-Direct (Pulmonary) or Indirect (Extra-pulmonary), as most experimental data suggest that these two entities have distinct pathogenic mechanisms.

b. Calculation of $\mathrm{PaO}_{2} / \mathrm{FiO}_{2}$ ratios with specific and standard ventilator settings (PEEP and MAP [mean airway pressure]). c. Exclude heart failure objectively (use of cardiac echocardiogram).

d. Only include patients with $\mathrm{P} / \mathrm{F}$ ratio with standard ventilator settings of less than $200^{3}$ (Table 3 ).

The first official description of ARDS was reported in 1967 and consisted of a case series of 12 patients with the acute onset of dyspnea, tachypnea, severe hypoxemia, chest radiographic abnormalities and decreased static respiratory system compliance. ${ }^{2}$ With the increased availability of pulmonary artery catheterization in ICUs, ARDS was recognized as a non-cardiogenic form of pulmonary edema, characterized by the accumulation of both protein and cells in the alveoli in the presence of normal left atrial pressures. Subsequently, several ARDS definitions were used in the early 1980s that required at least four basic clinical features, three of which were based on physiological and radiographic criteria adapted from this original case series:

\section{a. Hypoxemia (varying severity).}

b. Decreased respiratory system compliance.

c. Chest radiographic abnormalities (often of an ill-defined type and degree).

d. The fourth diagnostic criterion was usually the documentation of a pulmonary artery occlusion pressure of $18 \mathrm{mmHg}$ or less using a pulmonary artery catheter.

When the mortality associated with ARDS did not appear to improve during the 1980 s, the possibility was raised that the requirement of all four of these criteria biased the understanding of ARDS and contributed to the negative results of several therapeutic trials for ARDS. One concern was that these strict diagnostic criteria that required placement of a pulmonary artery catheter only identified patients with severe ARDS and a very poor prognosis. The requirement for pulmonary artery catheterization could also delay the diagnosis of ARDS [4]. Because approximately $50 \%$ of patients develop ARDS within the first 24 hours of meeting an at-risk diagnosis, delaying the administration of a therapy in order to insert a pulmonary artery catheter may diminish the chance for a successful therapeutic intervention. More recently, large clinical trials have demonstrated that a pulmonary artery catheter does not improve outcome for a variety of critically ill patients including patients with ARDS. The older diagnostic criteria of ARDS also lack specificity. For example, patients with vasculitis and alveolar hemorrhage meet the diagnostic criteria for ARDS, yet the pathogenesis of this disorder is different from ARDS. In addition, patients with an elevated pulmonary artery occlusion pressure are excluded, although these patients may have lung injury in addition to either hypervolemia or congestive heart failure (either systolic or diastolic). Several investigators postulated that the differences in reported epidemiological data, such as mortality rates, could be attributed to inconsistent cut off values for the hypoxemia criteria and variations in the interpretation of other diagnostic considerations. The presentation of ARDS includes a continuum of radiographic and arterial blood gas abnormalities, and any single cut off value for the definition of ARDS is arbitrary. Therefore, the identification of several gradations or categories of acute lung injury that have prognostic or therapeutic implications would be beneficial.

The murray lung injury score: Over the next two decades the basic definition was thought by many experts to be a hindrance to understanding the syndrome. The definition was not sufficiently specific, was open to varying interpretations, and did not require the clinical etiology of the syndrome to be specified. Investigators used different criteria to enrol patients in clinical studies making 
comparison of results across trials difficult. In 1988 Murray and colleagues proposed an expanded definition of ARDS intended to describe whether the syndrome was in an acute or chronic phase, the physiological severity of pulmonary injury, and the primary clinical disorder associated with the development of lung injury (Table 4). ${ }^{5,6}$ The definition can be analyzed as:

i. The first part of the definition addressed the clinical course separating acute from chronic cases; patients with a prolonged course (chronic) were presumably more likely to develop pulmonary fibrosis and to have poor outcomes.

ii. The lung injury score (LIS), quantified the severity of lung injury from the degree of arterial hypoxemia, the level of PEEP, the respiratory compliance, and the radiographic abnormalities (Table 5).

iii. The cause or associated medical condition must be specified. This proposal was accompanied by an editorial by Petty endorsing the new definition (Table $4 \& 5$ ).

The expanded definition had several advantages. By describing whether patients had an acute course with rapid resolution or a more chronic course, the definition differentiated between the rapidly resolving course typical of ARDS secondary to drug overdoses or pulmonary contusion and the complicated and protracted course of many patients with severe pneumonia or sepsis syndrome. The LIS quantified the severity of lung injury separating patients with severe lung injury (LIS $>2.5$ ) from those with mild lung injury (LIS $<2.5$ $>0.1$ ). Most importantly, the identification of the cause or associated medical condition addressed the etiology of lung injury. As the authors argued, [grouping all causes of ARDS under an umbrella classification potentially prevented the discovery of beneficial treatments aimed at a particular cause. ${ }^{6}$

The American-european consensus conference [AECC]: Subsequently, the American-European Consensus Conference (AECC) on ARDS was convened with a major goal of bringing uniformity to the definition of ARDS. The diagnostic criteria for ARDS proposed by this committee were $\mathrm{PaO}_{2} / \mathrm{FiO}_{2} \leq 200$, bilateral infiltrates on chest radiograph that need not be diffuse, and pulmonary artery occlusion pressure $\leq 18 \mathrm{mmHg}$ or no clinical evidence of left atrial hypertension when a pulmonary artery catheter was not used. The spectrum of disease severity was also expanded to include patients with milder hypoxemia. This definition, which includes patients with ARDS, was called acute lung injury (ALI), and the diagnostic criteria were similar except the $\mathrm{PaO}_{2} / \mathrm{FiO}_{2}$ ratio was $\leq 300$ (Table 6).

To determine the accuracy of the Murray lung injury score and the AECC definition, the diagnostic criteria for these two new definitions were compared to the previous definition of ARDS that required all four diagnostic criteria. Both definitions maintained a high degree of accuracy ( $>90 \%)$ for those ICU patients with a clearly defined at-risk diagnosis for the development of ARDS. Therefore, it is likely that the lung injury score and the AECC definition actually identify a patient population similar to the older strict definitions of ARDS for those patients with clearly defined at-risk diagnoses. The members of the AECC decided again to clarify some questions concerning their diagnostic criteria for ARDS. They acknowledged that the theoretical differentiation of ARDS from ALI based on severity of hypoxemia had not established two separate entities with different clinical associations and prognoses. In regard to the chest radiographic criteria, the committee stated that the bilateral infiltrates should be consistent with pulmonary edema, even if mild or patchy in nature. Opacities that were not considered appropriate for the radiographic criteria for ALI/ARDS include pleural effusions, pleural thickening, pulmonary masses or nodules, chronic scarring, volume loss, lobar collapse, plate-like atelectasis if the surrounding borders are sharp, extrathoracic opacities, and subcutaneous air. The AECC committee also commented on the difficulty in excluding hydrostatic or cardiogenic causes as the sole cause for pulmonary edema. They acknowledged the lack of a perfect cutoff value of the pulmonary artery occlusion pressure that would differentiate the hydrostatic pulmonary edema from permeability pulmonary edema (ARDS). However, no alterations in this controversial diagnostic criterion were recommended. The original AECC definition did not set a time limit for the word acute, but clearly ARDS needs to be differentiated from interstitial lung diseases that develop over weeks to months. Based on the results of one epidemiological study demonstrating that all patients developed ARDS by seven days of meeting the at-risk diagnosis, the length of time for the development of ARDS was defined as being less than seven days from the time of onset of their critical illness. In some patients with bilateral infiltrates, $\mathrm{PaO} / \mathrm{FiO}_{2}$ were lower than 200 while they received mechanical ventilation with zero or low levels of PEEP, but $\mathrm{PaO}_{2} / \mathrm{FiO}_{2}$ then increased to greater than 200 or even 300 when low to moderate levels of PEEP were subsequently applied. In these patients, atelectasis could have been an important cause of hypoxemia rather than shunt from consolidation and pulmonary edema. In one study the mortality of these patients was considerably lower than those whose $\mathrm{PaO}_{2} / \mathrm{FiO}_{2}$ remained below 200 after raising PEEP. Moreover, in many patients with bilateral infiltrates, $\mathrm{PaO}_{2} /$ $\mathrm{FiO}_{2}$ increased substantially when $\mathrm{FiO}_{2}$ were raised from moderate to high levels. This suggests that among patients with similar $\mathrm{PaO}_{2} /$ $\mathrm{FiO}_{2}$, oxygenation failure is worse and risk of death is higher in those receiving higher $\mathrm{FiO}_{2}$. Thus, without standardized or minimum PEEP and $\mathrm{FiO}_{2}$ criteria, the AECC criteria could identify a heterogeneous group of patients, some of whom are at low risk of adverse outcomes such as death. If so, then use of the AECC criteria to identify patients for ALI/ARDS trials, without PEEP and $\mathrm{FiO}_{2}$ criteria, could reduce the power of clinical trials because potential effects of new interventions may besmaller in patients with mild disease. Some investigators have speculated that the results of trials that enrolled ALI/ARDS patients using the AECC criteria without a minimum PEEP level were confounded by imbalances between study groups in patients with mild and severe lung injury that could not be detected without increasing PEEP. ${ }^{7}$ The National Institutes of Health ARDS Network used the AECC criteria as the inclusion criteria for most of its clinical trials since 1996. Exclusion criteria reduced heterogeneity of the enrolled populations, but patients were not excluded if they were on zero or low PEEP or low $\mathrm{FiO}_{2}$ [8]. However, it was found that the baseline PEEP alone predicted mortality, but after controlling for baseline $\mathrm{PaO}_{2} / \mathrm{FiO}_{2}$, PEEP did not predict mortality (Table 7). In conclusion the consensus conference definitions differed from the lung injury score in four areas:

a. The AECC definition did not use a four point chest $\mathrm{x}$ ray scoring system and instead looked for the appearance of bilateral infiltrates.

b. PEEP measurements were excluded in an effort to reduce variability in utilization of PEEP by individual physicians.

c. A different degree of hypoxemia was required for the oxygenation criteria.

d. They included either measurements of pulmonary artery occlusion pressure or absence of clinical evidence of left atrial hypertension. 
Anyhow, both scores identified the same group of patients despite differing criteria used in the definitions. The addition of PEEP requirement as a criterion in the lung injury score did not alter the sensitivity and specificity of the score, as shown previously by Moss [9]. The use of PEEP in restoring functional residual capacity in patients with acute lung injury ALI/ARDS is well accepted. Almost really all patients had a four point score from the chest $x$ ray value in the lung injury score, which would approximate with the chest radiograph finding of diffuse bilateral infiltrates in the AECC definition. All patients had a $\mathrm{PaO}_{2} / \mathrm{FiO}_{2}$ of $<175$, thus fulfilling both definitions of ARDS. Considerable discussion has been centered on the cut off between ALI and ARDS with regard to the $\mathrm{PaO}_{2} / \mathrm{FiO}_{2}$ with many suggesting a cut off value of $<150$ rather than $<200$. Concerns were that the more liberal definitions might include non-ARDS related illnesses with altered gas exchange. ${ }^{10}$

The Delphi definition of ARDS: In 2005, Ferguson and colleagues used a formal consensus-building process called the Delphi technique in an attempt to further improve upon the accuracy of the AECC definition of ARDS. The goal of this process was to reduce bias and improve the operating characteristics of the selected diagnostic criteria. A group of 11 opinion leaders first identified several defining characteristics of ARDS, and then refined and reduced these criteria through a pooled and anonymous feedback system. The end result were slightly different diagnostic criteria from the AECC definition including a definition of hypoxemia that required a $\mathrm{PaO}_{2} / \mathrm{FiO}_{2}$ ratio of $\leq 200 \mathrm{mmHg}$ with a PEEP of $\geq 10$, the documentation of a predisposing risk factor for the development of ARDS, and the reinsertion of a decreased static respiratory system compliance (Table 8).

Oxygenation index (OI) and $\mathrm{PaO}_{2} / \mathrm{FiO}_{2}$ ratio: Oxygenation Index (OI) is the most widely used system to quantify the degree of lung injury and hypoxemia in pediatric critical care. OI specifically takes into account mean airway pressure (MAP), an important determinant of oxygenation. OI is defined as the product of $\mathrm{MAP} \times \mathrm{FiO}_{2} \times 100 /$ $\mathrm{PaO}_{2}$. OI has been associated with outcome in both adults and children with ALI/ARDS. The original study in 2005 reported on the ability of OI to predict the duration of mechanical ventilation but not survival. Since then many adult studies have examined the efficacy of OI as a predictor of both duration of mechanical ventilation and mortality. In comparison, measurement of $\mathrm{PaO}_{2} / \mathrm{FiO}_{2}$ as a predictor of mortality in ALI/ARDS is uncertain. Although there are little differences in outcome based on $\mathrm{PaO}_{2} / \mathrm{FiO}_{2}$ ratio early in the course of ARDS, it is likely that persistently lower $\mathrm{PaO}_{2} / \mathrm{FiO}_{2}$ ratios are associated with higher mortality.

The Berlin definition: A proposal for an update of the AECC ARDS definition has been published recently by a task force panel of experts using a similar terminology as had been previously reported (ARDS definition task 2012). Using teleconferencing, in-person discussions and retrospective data, they proposed an ARDS classification with three severity categories (mild, moderate, and severe) for empirical evaluation. The term mild ARDS was used for defining those patients who are considered as ALI in the AECC definition $\left(300 \geq \mathrm{PaO}_{2} /\right.$ $\mathrm{FiO}_{2}>200 \mathrm{mmHg}$ ). The term moderate was used for patients with a $\mathrm{PaO}_{2} / \mathrm{FiO}_{2}>100 \mathrm{mmHg}$ but $<200$, and the term severe for those with $\mathrm{a} \mathrm{PaO}_{2} / \mathrm{FiO}_{2} \leq 100 \mathrm{mmHg}$. The panel had found that hospital mortality increased with every stage of severity [progression from one category to another is associated with increased mortality] (mild 27\%, moderate $32 \%$, severe $45 \%$ ) (Table 9). ${ }^{11}$

Although it is emphasized that the increased power of the new Berlin definition to predict mortality compared to the AECC definition, in truth it's still poor, with an area under the curve of only
0.577, compared to 0.536 for the old definition (Table 10). Clinical variables that are widely believed to be important and useful in the management of ARDS - static compliance of the respiratory system, radiographic severity, PEEP $>10$ and corrected expired volume $>10 \mathrm{~L} /$ min - were not predictive of mortality or other clinical outcomes. After including these variables in the initial draft definition and testing them empirically in the cohort, they were all dropped from the final Berlin definition for ARDS (Table 10).

It is really the authors did find a post-hoc high risk profile of patient with $52 \%$ mortality from ARDS. These patients had sever ARDS $\left(\mathrm{PaO}_{2} / \mathrm{FiO}_{2}\right.$ ratio $\left.<100\right)$ and either a static compliance of $\leq 20 \mathrm{ml} /$ $\mathrm{cmH}_{2} \mathrm{O}$ or a corrected expired volume of $\geq 13 \mathrm{~L} / \mathrm{min}$. What was wrong with old definition of ARDS:

a. Acute onset of hypoxemia with $\mathrm{PaO}_{2} / \mathrm{FiO}_{2}$ ratio $\leq 200 \mathrm{mmHg}$.

b. Bilateral infiltrates on chest $\mathrm{x}$ ray.

c. No evidence of left atrial hypertension.

In addition:

a. No clear criteria for defining acute - leading to ambiguity regarding cases of acuteon- chronic hypoxemia.

b. High inter observer variability in interpreting chest $\mathrm{x}$ rays.

c. Difficulties identifying/ruling out cardiogenic or hydrostatic pulmonary edema especially in an era of using pulmonary artery catheter.

d. $\mathrm{PaO}_{2} / \mathrm{FiO}_{2}$ ratio is sensitive to changes in ventilator settings.

The panel's findings sported by the European Society of Intensive Care Medicine, the American Thoracic Society (ATS) and the Society of Critical Care Medicine (SCCM) emerged from meetings in Berlin to try to address the limitations of the earlier AECC definition. ${ }^{12}$

Relationship between definitions: Several studies have examined the relationship between the definition of ARDS proposed by Murray et al and that of the NAECC. A prospective trial using strict diagnostic criteria for ARDS as the gold standard evaluated the diagnostic accuracy of the LIS, the NAECC definition, and a modified LIS in identifying patients with ARDS. The modified LIS consisted of two components: $\mathrm{a} \mathrm{PaO}_{2} / \mathrm{FiO}_{2}$ of $\leq 174$ (corresponding to grade 3 or higher LIS (Table 5)) and bilateral infiltrates on a chest radiograph. The following diagnostic criteria for ARDS served as the gold standard: concomitant presence of respiratory failure requiring mechanical ventilation; bilateral pulmonary infiltrates; $\mathrm{PaO}_{2} / \mathrm{PAO} 2<0.2$; $\mathrm{PAOP}<18 \mathrm{mmHg}$; and static respiratory system compliance $<50 \mathrm{ml} /$ $\mathrm{cm} \mathrm{H}_{2} \mathrm{O} .123$ patients with at least one of seven at risk diagnoses were followed prospectively for the development of ARDS. The diagnostic accuracy in the at risk population (true positive + true negative/total number of patients) was $90 \%$ for the LIS definition and $97 \%$ for both the modified LIS and the NAECC definitions. It is concluded that the three scoring systems identified similar populations when applied to patients with clearly defined at risk diagnoses.

A more recent study assessed the agreement between the definitions of Murray et al and the NAECC in diagnosing ARDS in a prospective trial of 118 patients comparing ventilation strategies. The incidence using the LIS was $62 \%$ while that using the AECC definition was $55 \%$. Statistical agreement between the two definitions was moderate and improved when analysis was limited to patients who had undergone compliance measurements (65\%). A more liberal $\mathrm{PaO}_{2} / \mathrm{FiO}_{2}$ ratio decreased agreement, as did increasing the LIS threshold diagnostic of ARDS to 3 or decreasing it to 2. Omitting 
data on oxygenation, chest radiography, PAOP, PEEP, or respiratory compliance either decreased agreement or left it unchanged. While agreement between the definitions was only moderate, there was no difference in mortality between the two groups of patients identified. The authors concluded that, for investigative purposes, the two criteria could be used interchangeably.

Significance of definitions in clinical trials: The AECC updated its recommendations in 1998. Although no formal changes were made, the Committee emphasized the importance of addressing epidemiological and etiological differences between patients when designing clinical trials. Several prospective studies had identified risk factors present at the onset of lung injury that predicted poorer outcomes; clinical trial organizers would need to ensure an equal distribution of these risk factors in their experimental and control arms. They also encouraged further research focused on identifying markers predictive of progression to or poor outcomes from lung injury. Previous definitions had relied on abnormalities of lung physiology to grade injury; however, neither the initial LIS nor the initial $\mathrm{PaO}_{2} / \mathrm{FiO}_{2}$ ratio was predictive of mortality in clinical trials. Some biological markers, on the other hand, had already been proved to be useful in identifying patients at risk for poor outcomes. For example, raised levels of procollagen III peptide in early broncho alveolar lavage and pulmonary edema fluid samples predicted a protracted clinical course with progression to pulmonary fibrosis in patients with ALI/ARDS, and increased levels of von Willebrand factor antigen in plasma predicted the development of lung injury in patients with non pulmonary sepsis syndrome. Also, a recent study reported that higher levels of von Willebrand factor antigens in the plasma of ALI/ARDS patients independently predicted mortality early in the clinical course. The importance of standard definitions and a mechanistic approach to enrolling patients in clinical trials are apparent in the results of two recent randomized multicentre trials. The first, conducted by the ARDS Network, evaluated the benefits of a low tidal volume strategy of mechanical ventilation and found an absolute reduction in the primary outcome of death prior to hospital discharge of $9 \%$ using lower tidal volumes (22\% relative reduction in mortality). Patients were enrolled using the 1994 definition and the benefit of a protective ventilation strategy was maintained in all subsets of patients with ALI/ARDS.

The importance of identifying the mechanism of lung injury is borne out by a recent trial of recombinant human activated protein $\mathrm{C}$ in severe sepsis. A 96 hour infusion of protein $\mathrm{C}$ resulted in an absolute reduction in mortality of $6.1 \%$ (20\% relative reduction) in a large international multicentre trial; $54 \%$ of the 1690 patients had a pulmonary source of sepsis and $75 \%$ required mechanical ventilation on entry into the study. Although the study did not report the number of patients who met the criteria for ALI/ARDS, it is likely that most did since ARDS is the most common cause of respiratory failure in sepsis and sepsis is the most common predisposing factor for the development of ARDS. Based on this study, it is likely that a trial of protein $\mathrm{C}$ in patients with lung injury due to sepsis will show a treatment benefit while the same trial in patients with ARDS due to trauma or fat emboli may not. As more is learned about the epidemiology and pathophysiology of ARDS, identifying the cause of injury in designing treatment trials will become increasingly critical. ${ }^{6}$

Persistent problems and controversies involving the definition of ARDS: In spite of these several attempts to develop diagnostic criteria for ARDS, fundamental problems still remain. Esteban and colleagues determined the validity of the AECC diagnostic criteria against autopsy results in 382 patients who died while in the ICU. Patients were considered to fulfil the pathological diagnosis of ARDS if they had diffuse alveolar damage defined as the presence of hyaline membranes plus at least one of the following criteria: alveolar type I or endothelial cell necrosis, edema, organizing interstitial fibrosis, or prominent alveolar type II cell proliferation. A total of 127 (33\%) of the patients met the AECC diagnostic criteria, and 112 (29\%) met the pathological diagnosis. The sensitivity and specificity of the AECC definition using the pathological diagnosis as the gold standard was $75 \%$ and $84 \%$, respectively. The accuracy of the AECC definition did not improve when only the 284 patients with risk factors were included. The most common pathological findings in those patients who met the AECC criteria for ARDS but did not fulfill the pathological diagnosis of ARDS were pneumonia, pulmonary hemorrhage, and pulmonary edema. Conversely, the clinical diagnoses of the patients who met the pathological criteria for ARDS but did not meet the AECC clinical criteria were again pneumonia and pulmonary edema. A Brazilian study examining the results of only 22 autopsy studies again confirmed the relative inaccuracy of the AECC definition. ${ }^{13}$ Studying a subset of 138 patients from the previously cited Esteban data, the diagnostic accuracy of the lung injury score and the recently developed Delphi definition were determined and compared to the AECC diagnostic criteria. Unfortunately, these two additional definitions of ARDS also did not accurately identify patients with pathological evidence of ARDS. The sensitivity for the diagnosis of ARDS was similar for all three definitions. However, the specificity of the lung injury score and the Delphi definition were significantly better than the AECC definition.

The chest radiographic criteria used to define ARDS also remain problematic. When 21 established investigators reviewed 28 randomly selected chest radiographs from intubated patients with a $\mathrm{PaO}_{2} / \mathrm{FiO}_{2}$ of $<300$, there was considerable variability in their interpretations of whether the films were consistent with ARDS. Other studies have also demonstrated a similar lack of consistency in chest radiographs scores when using the four-point radiographic criteria from the Murray lung injury score. However, formal training in the interpretation of chest radiographs for the diagnosis of ARDS can improve the inter-rater reliability between investigators. After a standardized session during which physicians refined the standards and rules they would apply to the radiographic interpretation, their inter-rater reliability in regard to identification of chest radiographs consistent with ARDS was nearly perfect. Therefore, formal training in radiographic interpretation among investigators would decrease the heterogeneity of patients enrolled in clinical trials for ARDS. There is also controversy surrounding the specific hypoxemia criteria included in the definition of ARDS. Individual or institutional variations in mechanical ventilator strategy could impact the incidence of ARDS/ ALI in an ICU or medical center. Increasing the level of PEEP alone may improve the $\mathrm{PaO}_{2}$ and directly alter the $\mathrm{PaO}_{2} / \mathrm{FiO}_{2}$ ratio. Several studies have demonstrated that up to $50 \%$ of patients who meet the hypoxemia criteria for ARDS without PEEP will significantly improve their oxygenation with higher levels of PEEP, and subsequently may no longer meet the hypoxemia criteria for ARDS. As a result, a PEEP requirement of $12 \mathrm{~cm} \mathrm{H}_{2} \mathrm{O}$ was included in the hypoxemia criteria in the Delphi definition in order to exclude these patients as being diagnosed with ARDS. However, it is presently unclear what specific level of PEEP should be included in the definition and whether the inclusion of a PEEP requirement results in the identification of a more homogeneous group of ARDS patients. Anyhow, in order to differentiate ARDS from hydrostatic (cardiogenic/volume overload) pulmonary edema, the AECC consensus definition includes either a pulmonary artery occlusion pressure of $\leq 18 \mathrm{mmHg}$ or no clinical evidence of left atrial hypertension. Some patients who meet clinical criteria for ARDS may have a pulmonary capillary occlusion pressure 
that is above the selected cut off value of $18 \mathrm{mmHg}$. In the ARDS network pulmonary artery catheter trial, $29 \%$ of ALI patients had a pulmonary artery occlusion pressures that exceeded the traditionally accepted threshold of $18 \mathrm{mmHg}$. In addition, there are no specific recommendations by the AECC committee about what constitutes clinical signs of an elevated left atrial pressure. Depending on the specific diagnostic criteria used to define clinical left atrial hypertension, between $5 \%$ and $30 \%$ of patients meeting chest radiograph and hypoxemia criteria may be excluded from a formal diagnosis of ARDS. Despite these concerns, the present diagnostic criteria of the AECC definition have proven to be extremely useful in regar to clinical trial design. Importantly, patients who meet the present AECC definition of ARDS respond to specific therapies that are associated with improvement of their outcome including a $25 \%$ reduction in mortality with the use of a low tidal volume ventilatory strategy and a significant reduction in ventilator free days with the use of a fluid conservative strategy. ${ }^{1}$

\section{Accuracy of Clinical diagnosis of acute respiratory distress syndrome}

Using the AECC criteria, and consequently the accuracy limitations, other authors conducted a regional study in the USA and found that the incidence of acute lung injury (including ARDS and less serious levels of hypoxemia, but with the other findings of the syndrome) was 78.9 for every 100,000 inhabitants. ${ }^{14}$ Mortality data are also limited. Most studies are conducted in specific patient groups with strict inclusion and exclusion criteria, with the objective of assessing treatment strategies rather than mortality. Among these, two more recent studies reported mortality rates of $35 \%$ and $40 \%$. The epidemiologic trials conducted by these two groups of authors produced similar outcomes, both reporting an ARDS mortality rate of $41.1 \%$. However, these numbers demonstrate the importance of recognizing ARDS, and the need for a reliable clinical definition, since an accurate diagnosis, obtained from histopathological findings, is not possible in most cases. The principal finding of many studies was that the sensitivity and specificity of the AECC criteria to diagnose ARDS, which are widely used in everyday practice and to conduct clinical trials, are not appropriate. Consequently, the positive and negative likelihood ratios obtained cannot safely confirm or rule out, respectively, a diagnosis of ARDS. Further, in a tailored study that also evaluated the efficiency of the AECC criteria based on autopsy results, another group of authors found similar values. Of 127 patients with clinical ARDS criteria, 43 did not present histopathological findings of diffuse alveolar damage. The principal findings for these patients were: pneumonia, alveolar hemorrhage, cardiogenic pulmonary edema, pulmonary embolism and pulmonary interstitial disease secondary to chemotherapy. In contrast, the autopsy confirmed the presence of diffuse alveolar damage in the 27 patients that did not present clinical criteria for the ARDS diagnosis. Of those 27,12 had been diagnosed with pneumonia, 12 had been diagnosed with cardiogenic pulmonary edema, and 3 had not been diagnosed with any respiratory disorder. These discrepancies between clinical and histopathological findings in relation to ARDS reveal the limited accuracy of the AECC criteria, which were found to have a sensitivity of $75 \%$, a specificity of $84 \%$, a positive likelihood ratio of 4.7 and a negative likelihood ratio of 0.3 .

The reason for this low accuracy is that, although ARDS is a complex inflammatory condition that attacks the alveolar-capillary barrier, involving various mediators and the development of edema and alveolar collapse, the AECC diagnostic criteria only reflect the consequences of these alterations on the chest $\mathrm{x}$ ray and gas exchange, as well as attempting to ignore the cardiogenic nature of the process. With such great discrepancies, and in view of the fact that the lungs suffer diverse types of injuries related to various etiologies, it is not surprising that the lung injury diagnosis criteria are not accurate. However, there is no simple solution for this problem, and this could explain the fact that the AECC criteria have continued to be used since their implementation in 1994, despite the known limitations, to clinically diagnose ARDS. For example, adding inflammatory markers to the diagnosis in an attempt to include pathogenic data would not necessarily increase the accuracy, since there are many mediators involved and none are specific to the syndrome. In addition, invasive procedures such as bronchoalveolar lavage, which is not always easy to perform in critical patients, would be required to measure the markers involved in the lung injury. The primary implication of these results is that when there is a clinical suspicion of ARDS, other diagnostic hypotheses should be considered, whether associated with the syndrome or not. There is a diverse group of pulmonary parenchymal diseases with non infectious etiologies such as alveolar hemorrhage, pulmonary embolism, neoplasias, bronchiolitis obliterans organizing pneumonia, acute eosinophilic pneumonia and drug toxicity that could have acute onset and meet all the clinical, physiological and radiographic criteria for ARDS. Other diseases with infectious etiologies could also simulate the syndrome. In 2004, one group of authors published a study with the objective of evaluating the role of the lung biopsy in ARDS. In that study, involving 57 patients, the most common alternative or concomitant diagnosis to ARDS were infection in 8 cases, alveolar hemorrhage in 5 cases and bronchiolitis obliterans organizing pneumonia in 5 cases, as well as drug reactions, lymphangitic carcinomatosis, chronic eosinophilic pneumonia, allergic bronchopulmonary aspergillosis and cardiogenic pulmonary edema in 1 case each. Based on the results, the treatment for most of the patients was changed, with the addition of specifictherapy in $60 \%$ and the discontinuation of unnecessary treatment in $37 \%$. Another significant implication of these results is that it is possible that not all of the patients involved in the studies investigating different ARDS treatment strategies, whether using ventilators or medication, really had the disease. These studies typically use AECC criteria to define ARDS, which, as demonstrated by our results and those of other authors, have limited accuracy. Therefore, positive or negative evidence regarding different ARDS treatments could be determined based on unreliable criteria. This limitation could be minimized by establishing stricter inclusion criteria beyond the simple clinical diagnosis of ARDS. Some authors already limit inclusion in studies of ARDS ventilator strategies to patients that continue to present radiographic alterations and hypoxemia consistent with ARDS after a minimum positive end-expiratory pressure of, for example, $10 \mathrm{~cm}$ $\mathrm{H}_{2} \mathrm{O}$ has been applied for at least 24 hours. Even if this type of strategy does not improve diagnostic accuracy, it establishes a more restrictive level of lung injury severity, increasing the reproducibility of the results obtained. ${ }^{13}$

\section{The differences between Pulmonary and Extrapulmonary acute respiratory distress syndrome}

Since its initial description, the acute respiratory distress syndrome has been considered as a morphological and functional expression of a similar underlying lung injury caused by a variety of insults. In fact, Ashbaugh et al ${ }^{2}$ in defining this syndrome stated that "The etiology of this respiratory distress syndrome remains obscure. Despite a variety of physical and possibly biochemical insults, the response of the lung was similar in all 12 patients. In view of the similar response of the lung to a variety of stimuli, a common mechanism of injury may be postulated". ${ }^{2}$ This observation used the term syndrome to refer to "a 
group of symptoms and signs of disordered function related to one another by means of some anatomic, physiologic, or biochemical peculiarity". In 1994, the American-European Consensus Conference defined two pathogenetic pathways leading to ARDS: a direct (primary or pulmonary) insult that directly affects lung parenchyma and an indirect (secondary or extrapulmonary) insult that results from an acute systemic inflammatory response. The differentiation between direct and indirect insult is often straightforward as for primary diffuse pneumonia or ARDS originating from intra-abdominal sepsis. In other situations, the precise identification of the pathogenetic pathway is somewhat questionable, as for trauma or cardiac surgery. The distinction, however, was mainly speculative until Gattinoni et al. reported possible differences in the underlying pathology, respiratory mechanics, and response to PEEP in pulmonary ARDS (primarily pneumonia) and extrapulmonary ARDS (primarily due to abdominal disease). In (Table 11) are reported the some of the underlying etiologies in pulmonary ARDS and extrapulmonary ARDS. ARDS occurs following a variety of risk factors. Strong evidence that supports a causeand- effect relationship between ARDS and risk factors was identified for sepsis, trauma, multiple transfusions, aspiration of gastric contents, pulmonary contusion, pneumonia, and smoke inhalation. However, only a few studies have investigated the prevalence and mortality considering pulmonary ARDS and extrapulmonary ARDS In the majority of available studies the prevalence of pulmonary ARDS was higher compared to extrapulmonary ARDS, varying from 47 to $75 \%$ of all cases. In the most recent retrospective analysis of patients enrolled in the Acute Respiratory Distress Syndrome Network (ARDSNet) trial of low tidal volume ventilation, roughly an equal proportion of pulmonary ARDS and extrapulmonary ARDS were identified. It has been reported that pulmonary trauma was associated with higher survival rate, whereas opportunistic pneumonia had a lower survival rate. Among complications, acute renal failure, pulmonary infection, and bacteraemia seem to be independent factors associated with increased mortality. However, the reported mortality in patients with ARDS attributable to pulmonary and extrapulmonary causes varies considerably. In one study a direct pulmonary insult triggering ARDS was identified as being associated with increased mortality, whereas in another study no relationship was found between direct pulmonary insults and increased mortality (36\% and 34\% mortality for pulmonary and extrapulmonary causes, respectively). Moreover, in the same cohort of patients, the proportion of patients in whom organ failure developed the pulmonary and extrapulmonary was equal between groups, and the proportion achieving liberation from mechanical ventilation at 28 days was also identical. The lack of agreement among various studies can be explained by differences in:

\section{a. Baseline status.}

b. The prevalence of the disease precipitating ARDS in each Centre.

\section{c. The impact of therapy.}

\section{d. The overall distribution of these factors in the studied population}

Thus, it is not known whether different clinical management and ventilatory treatment modified accordingly with the different pathophysiological characteristics could improve outcome (Table 11). The alveolar-capillary barrier is formed by two different structures, the alveolar epithelium and the vascular endothelium. Traditionally, it has been though that insults applied to the lung, through the airways or the circulation, result in diffuse alveolar damage. Although many insults may converge in the stage of ARDS, it is really wonder if, in early stages, a direct or indirect insult to the lung may have different manifestations. The different histological and biochemical alterations in pulmonary ARDS and extrapulmonary ARDS are reported in (Table 12).

A direct insult has been studied in experimental models by using intratracheal instillation of endotoxin, complement, tumor necrosis factor (TNF), or bacteria. After a direct insult, the primary structure injured is the alveolar epithelium, while the capillary endothelium is roughly normal. This causes activation of alveolar macrophages and neutrophils and of the inflammatory network, leading to intrapulmonary inflammation. An increased amount of apoptotic neutrophils and altered type I and type II cells has been reported as well as an increase in interleukins (IL)-6, 8 and 10 in the bronchoalveolar lavage (BAL) in direct injury compared to indirect injury. The prevalence of the epithelial damage determines a localization of the pathological abnormality in the intra-alveolar space, with alveolar filling by edema, fibrinous exudate, collagen, neutrophilic aggregates, and/ or blood, with a minimum interstitial edema. This pattern has often been described as pulmonary consolidation, probably representing a combination of alveolar collapse and prevalent fibrinous exudates and alveolar wall edema in pulmonary ARDS.

An indirect insult has been studied in experimental models by intravenous or intra peritoneal toxic injection. After an indirect insult, the lung injury originates from the action of inflammatory mediators released from extra pulmonary foci into the systemic circulation. In this case, the first target of damage is the pulmonary vascular endothelium, with an increase of vascular permeability and interstitial edema. A decreased amount of apoptotic cells has been described in experimental model of extra pulmonary ARDS as well as a decreased amount of ILs in the BAL. Thus, the pathological alteration due to an indirect insult is primarily micro vascular congestion and interstitial edema, with relative sparing of the intra-alveolar spaces. Histologically the ARDS lung is characterized by diffuse lung damage with subdivision of temporal course in early and late lesions, designated as acute and chronic fibro proliferative diffuse alveolar damage. The acute stage of diffuse lung damage by interstitial and intra alveolar edema and hyaline membrane. This stage is followed by consecutive proliferation by fibroblastic cells characterized by chronic and or fibro proliferative damage. The disease process finally leads to the gross destruction of the pulmonary lobes resulting in fibrosis and honeycombing. A recent some studies have described the morphological differences between pulmonary lesions in patients with pulmonary ARDS and extra pulmonary ARDS. They found a predominance of alveolar collapse, fibrinous exudate and alveolar wall edema in pulmonary ARDS. However the acute inflammatory phase of lung injury is also associated with fibro proliferative response that leads to alveoli obliteration and derangement in the spatial distribution of the extracellular matrix. Also it is recently reported an increased collagen content in pulmonary ARDS than in extra pulmonary ARDS in the early phase of the disease, while no differences were observed concerning the elastic fibers ontent. It is therefore concluded that extracellular matrix remodeling occurs early in the development of ARDS and appears to depend on the site of the initial insult, being prevalent in pulmonary ARDS. In general these experimental and in vivo findings suggest that the damage in the early stage of direct insult is primarily focused on the alveolar epithelium, whereas in indirect injury on the vascular endothelium. The inflammatory agents are more increased in the serum in extra pulmonary ARDS, while in the BAL in pulmonary ARDS. However, it is worth noting the possible co-existence of the two insults: one lung with direct injury (as pneumonia) and the other with indirect injury (through mediator release from the original pneumonia). ${ }^{15}$ 
In recent years, a number of studies have identified differences by computed tomography (CT) between pulmonary ARDS and extra pulmonary ARDS. It is found that by performing three representative scans at the apex (top of the upper aortic arch), at the hilum (first section below the carina), and at the base $(2 \mathrm{~cm}$ above the highest diaphragm). The entilatory setting was not standardized during scans. The lung was scored as follows: "normal lung," "ground-glass opacification" (mild increased attenuation with visible vessels), and "consolidation" (markedly increased attenuation with no visible vessels). It is found that in extra pulmonary ARDS, ground-glass opacification was more than twice as extensive as consolidation (Figure 1A \& B). This contrasted markedly with pulmonary ARDS, in which there was an even balance between ground-glass opacification and consolidation. When the type of opacification between the two groups was compared, the patients with extra pulmonary ARDS had $40 \%$ more ground-glass opacification than did those with pulmonary ARDS. Conversely, the pulmonary ARDS patients had $>50 \%$ more consolidation than did those with extra pulmonary ARDS. It is also found differences in the regional distribution of the densities. In extra pulmonary ARDS ground-glass opacification was greater in the central (hilar) third of the lung than in the sternal or vertebral third. There was no significant craniocaudal predominance for ground-glass opacification or consolidation, but consolidation showed a preference for the vertebral position over the sternal and central positions. In extra pulmonary ARDS ground-glass opacification was evenly distributed in both the craniocaudal and sterna vertebral directions. Consolidation tended to favor the middle and basal levels, but also favored the vertebral position. The total lung disease was almost evenly distributed between the left and right lungs in both pulmonary ARDS and extra pulmonary ARDS. However, grossly asymmetric disease was always due to asymmetric consolidation. Moreover, the presence of air bronchograms and pneumomediastinum were prevalent in pulmonary ARDS, while emphysema-like lesions (bullae) were comparable in both types of ARDS (Figure 1A \& B).

It was reported that a significantly higher incidence of intense parenchymal opacification in nondependent areas of the lung in patients with direct insults (indicative of consolidation secondary to inflammatory infiltrate), but no other differences emerged between the two types of patients. Moreover, the extent of intense parenchymal opacification in nondependent areas of the lung was inversely related to the time from intubation to $\mathrm{CT}$. It was concluded that differentiating between pulmonary ARDS and extra pulmonary ARDS on the basis of CT findings is not straightforward, and that no single radiological feature is specifically associated with lung injury of either type. It is currently found that all the patients with ARDS showed consolidation opacities in the dependent part of the lung (Figure 2A). However differently from pulmonary ARDS originating from communityacquired pneumonia, in ventilator-associated pneumonia the amount of aerated lung was increased while ground-glass opacification was less compared to community-acquired pneumonia. However, when these patients were turned prone a marked reduction of previously dependent densities was found (nondependent in prone, (Figure 2B). This suggests that lung areas previously considered consolidated due to ventilator associated pneumonia, were not really consolidated but mainly atelectatic. ${ }^{16}$ Application of recruitment maneuvers or PEEP (up to $15 \mathrm{cmH}_{2} \mathrm{O}$ ) were unsuccessful to reopen these zones in supine position, likely because of a marked in homogeneity of pulmonary parenchyma (well aerated-elastic in nondependent and non-aeratedstiff in dependent zones). Thus, it is possible to hypothesize that the pathophysiology and the lung morphology in pulmonary ARDS may be different in community-acquired pneumonia and ventilatorassociated pneumonia. It is possible that the period of time from the infection and the development of severe respiratory failure (usually within 1 week), can favor some initial diffusion of inflammatory agents, which can explain the presence of amounts of groundglass opacification in pulmonary ARDS from community-acquired pneumonia. Moreover, the aggressive therapeutic management ventilator-associated pneumonia, and thus a potential reduction in release of inflammatory agents in the peripheral circulation, may limit the radiological pattern to consolidation and/or atelectasis.

However, with all the limits and somewhat arbitrary classification of patients and interpretation of morphological observation, these findings support the hypothesis that the radiological pattern is different in pulmonary ARDS and extra pulmonary ARDS. It can be concluded that:

i. In ARDS, the increase in the lung densities is most prominent in the dependent lung regions in the supine position but may, in a minority of patients, be more homogeneously distributed throughout the lung parenchyma.

ii. In pulmonary ARDS due to community-acquired pneumonia, two prevalent patterns have been described: the first, extensive consolidation and air bronchograms in the dependent part of the lung together with ground-glass opacification, or the second, homogeneous diffuse interstitial and alveolar infiltration, without evidence of atelectasis.

iii. In pulmonary ARDS, due to ventilator-associated pneumonia, densities in the dependent part of the lung (likely atelectasis) are prevalent with the remaining nondependent lung substantially normal.

iv. On the contrary in extra pulmonary ARDS, there is predominantly ground-glass opacification.

Traditionally, the mechanical alterations of the respiratory system observed during ARDS were attributed to the lung because the chest wall elastance was considered nearly normal. Studies in which respiratory system, lung, and chest wall mechanics were partitioned have proved this assumption wrong. The present investigations consistently found that the elastance of the respiratory system was similar in pulmonary ARDS and extra pulmonary ARDS, but the elastance of the lung was higher in pulmonary ARDS, indicating a stiffer lung. Conversely, the elastance of the chest wall was more than 2 folds higher in extra pulmonary ARDS than in pulmonary ARDS, indicating a stiffer chest wall. The increase in the elastance of the chest wall was related to an increase in the intra-abdominal pressure, which was 3 folds greater in extra pulmonary ARDS. In critically ill patients, data on intra abdominal pressure are surprisingly scanty. In most of the current studies patients, the elevated values could be explained by primary abdominal disease or edema of the gastrointestinal tract. The sonographic findings of the abdomen were analyzed in normal spontaneously breathing subjects, in patients with extra pulmonary ARDS due to abdominal sepsis, and in patients with pulmonary ARDS due to community-acquired pneumonia. In the normal subjects it was difficult to recognize the abdominal wall and the gut anatomical structure. In the patients with extra pulmonary ARDS and related abdominal problems, the increased dimension and thickness of the gut, with intraluminal debris and fluid and with reduced peristaltic movements, were visible. In the patients with pulmonary ARDS, the dimension of the gut were slightly increased while the gut wall thickness was not increased, without any consistent debris or fluid. Thus, it is evident that patients with abdominal problems present important anatomical alterations of the gut, which can explain the increased intra-abdominal pressure. Thus, these findings suggest that 
in ARDS the increased elastance of the respiratory system is produced by two different mechanisms: in pulmonary ARDS a high elastance of the lung is the major component, whereas in extra pulmonary ARDS increased elastance of the lung and of the chest wall equally contributed to the high elastance of the respiratory system. Moreover, it was found that respiratory resistance, partitioned into its airway and viscoelastic components, was comparable in pulmonary ARDS and an extra pulmonary ARDS. However, the resistance of the chest wall was also elevated in extra pulmonary ARDS and significantly correlated to intra-abdominal pressure, suggesting that intra-abdominal pressure can affect the viscoelastic properties of the thoraco abdominal region.

However, it is important to consider that most of the patients in the extra pulmonary ARDS had ARDS caused by intra-abdominal pathological conditions, and it seems likely that some of the changes seen in chest wall elastance relate to intra-abdominal mechanics and effects on diaphragmatic movements. Altered lung elastance with relatively normal chest wall elastance was also found in patients affected by severe P. carinii pneumonia, and in patients with ventilatorassociated pneumonia that usually present the same histopathology as pulmonary ARDS. ${ }^{17}$

\section{Gender and acute respiratory distress syndrome}

The Acute Respiratory Distress Syndrome remains a considerable contributor tomorbidity and mortality after critical injury. Several authors have hypothesized gender as accounting for differences in outcomes following trauma, particularly in animal models. It was hypothesized that the pro-inflammatory properties of estrogens would predispose females to developing ARDS (Figure 3). Gender differences in the prevalence of asthma are well described with females being at a higher risk. For instance in a recent analysis of patients hospitalized for an asthma exacerbation in England, women had a higher death rate. Female gender has also been identified as a risk factor for hospitalization in COPD patients. Recent data adds support to the hypothesis that the female lung is more prone to injury and the deleterious effects of inflammation. The effect of female gender on the development of ARDS was independent of the older age and differing injury patterns in these patients. It is found that despite higher rates of ARDS, females with ARDS did not die more frequently than their male counterparts with ARDS. The fact that females are more prone to ARDS following trauma supports the hypothesis that sex hormones may contribute to the propensity to develop lung injury.

Estrogens have diverse and extensive immunologic properties, giving plausibility to the fact that the pro-estrous state would be associated with exaggerated inflammation and ARDS. Numerous data confirms that higher levels of endogenous estrogens are associated with higher rates of ARDS in both genders. However, these differences do not fully explain the difference in ARDS rates by sex since females have a higher incidence of ARDS at all estradiol levels than males (Figure 3).

There is similar biologic plausibility for the association between lower testosterone and higher rates of ARDS since testosterone is generally considered immune depressing. Also, global hypothalamicpituitary-gonadal axis suppression, and therefore, testosterone depression is related to illness severity. Many data confirms this relationship in both genders. Anyhow, it was concluded that females are more likely than males to develop ARDS following critical injury. However, regarding the concept of sex hormones, specifically estrogens, having immunologic properties enabling the development of lung injury. For both male and female patients, ARDS increases in incidence with increasing estradiol levels. Additionally, for both male and female patients, ARDS declines with increasing testosterone levels. ${ }^{18}$ Despite the increased incidence in ARDS, the mortality in patients with ARDS does not differ according to gender. These findings are not fully explained by sex hormones, but there is evidence that the relationship between pro-inflammatory estrogens and immune depressing testosterone and development of ARDS are important regardless of gender. ${ }^{19}$

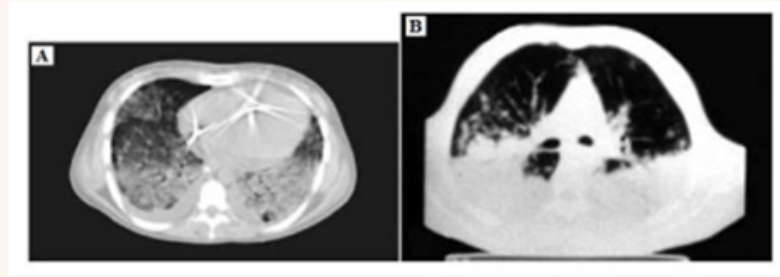

Figure I A computed tomography scan of extra pulmonary acute respiratory distress syndrome at end expiration. There is a predominantly ground-glass Opacification.

B A computed tomography scan of pulmonary acute respiratory distress syndrome at end-expiration. There is extensive consolidation, with an approximately equal amount of normal lung and ground-glass opacification and air bronchograms.

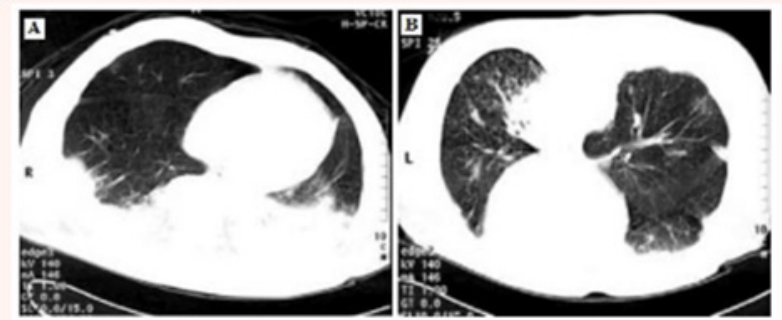

Figure 2 A computed tomography scan of pulmonary acute respiratory distress syndrome due to ventilator associated pneumonia at end-expiration.

A. Supine position with extensive bilateral apparent consolidations;

B. Prone position, with an almost total clearing of the apparent consolidations. This indicates the atelectatic nature of the densities.

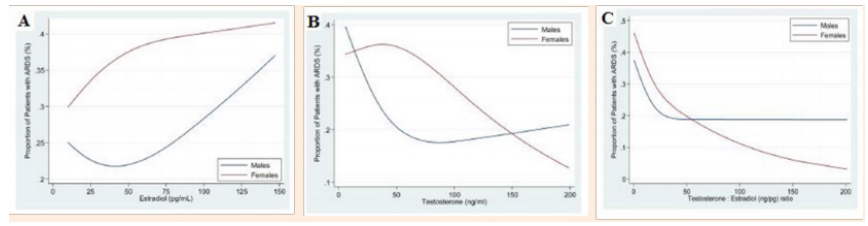

Figure 3 The relationship between sex hormones and ARDS stratified by gender.

A. Demonstrates that for both genders, rates of ARDS increase with increasing estradiol. The higher rates of ARDS observed in women are not fully explained by estrogens, however, since for all estradiol levels, the rate of ARDS is higher in females.

B. Demonstrates that for both genders, rates of ARDS decrease with increasing testosterone.

C. Demonstrates the relationship between testosterone and estradiol (testosterone to estradiol ratio) and rates of ARDS. For both genders a higher ratio appears protective, with the lowest rates of ARDS being expected in patients with ratios greater than 50 .

\section{Recurrent acute lung injury}

With the improvement of ALI survival in population it was observed that a significant number of patients who had more than one episode of ALI (recurrent ALI). Gastroesophageal reflex disease 
was identified as the most important risk factor for recurrent ALI, potentially suggesting an important role of gastric aspiration in the development of this syndrome. ALI represents a specific injury pattern resulting from a variety of insults in a susceptible host. Aspiration is an important recognized mechanism for acute lung injury found to be a risk factor in $11 \%$ with an associated mortality of $44 \%$. Unfortunately, aspirations in both clinical practice and in research are limited by its clinical definition of a witnessed aspiration event, raising the concern that many aspiration events that lead to ALI/ARDS are unrecognized. In those with witnessed gastric aspiration, it observed a dose dependent pattern from $5 \%$ in those who had no ALI, to $16 \%$ in those with a single episode of ALI, to finally $37 \%$ in those who had recurrent ALI (Tables $13 \& 14$ ). With a lack of a proven standard for identifying gastric to pulmonary aspiration, it was looked for other signals that may indicate that silent aspiration occurs more frequently as a cause of ALI and ARDS. Mechanistically, for gastric to pulmonary aspiration to occur, there must first be reflux of gastric contents. This may occur acutely as can be seen in the critically ill (e.g. emesis and aspiration in a semiconscious or unconscious patients), but more commonly it is seen in the chronic condition of gastro-esophageal reflux disease. Gastroesophageal reflex disease could act as the necessary prerequisite risk for aspiration, and furthermore, because it is chronic, it may be a risk for repeated episodes of silent aspiration and acute lung injury. With a prevalence of gastro-esophageal reflux disease of $20 \%$, the atrisk population therefore, may be highly significant. Notably, the risk for ALI as a result of having gastro-esophageal reflux disease was higher than for aspiration itself. However, this is not unexpected as aspiration is often insensitively defined clinically, and suggests that potentially, many aspiration events are unrecognized. This highlights the very important need for a more objective determination of aspiration. It was noticed that the chronic use of acid suppressive medications did not alter the susceptibility of ALI development. In the critically ill, acid suppression is typically utilized for the prevention of upper intestinal bleeding. There is increasing concern as to whether the empiric use of potent acid suppression may increase the risk for pneumonia and clostridium difficile infection. However, there is no data on how acid suppression might modify the risk for ALI, whether favorably or unfavorably. Although it was expect the acid suppression would confer a protective effect, its concomitant increase in risk for pneumonia and subsequent lung injury may be unfavorable. However, it was found that the incidence of recurrent ALI was about 2.02 per 100,000 person/years. When affected with a second episode of ALI, mortality was high at $47 \%$, with a median survival of about 22 days ${ }^{20}$ (Table 13, 14).

\section{Clinical risk factors for acute lung injury and acute respiratory distress syndrome}

Most cases of ALI/ARDS in adults are associated with pulmonary sepsis $(46 \%)$ or non pulmonary sepsis $(33 \%)$. Risk factors include those causing direct lung injury (e.g., pneumonia, inhalation injury, pulmonary contusion) and those causing indirect lung injuries (e.g., non pulmonary sepsis, burns, transfusion-related acute lung injury). Risk factors in children are similar to those in adults, with the addition of age-specific disorders, such as respiratory syncytial virus infection and near drowning aspiration injury. Anyhow, (Table 15) includes signs and symptoms suggesting specific causes of ARDS. (Table 15).

Table I 3 Association with recurrent $A L I$ and single $A L I^{5}$

\begin{tabular}{|c|c|c|c|c|c|c|}
\hline \multirow[b]{2}{*}{ Variables } & \multicolumn{2}{|c|}{ Exposure frequency } & \multicolumn{2}{|c|}{ Discordant pairs* } & \multicolumn{2}{|c|}{ Concordant pairs* } \\
\hline & Recurrent ALI\% & Single ALI\% & $+1-$ & ++ & $+/+$ & + \\
\hline Gastro-esophageal reflex disease & 79 & 42 & 7 & 0 & 8 & 4 \\
\hline Proton pump inhibitors use & 42 & 42 & 6 & 6 & 2 & 5 \\
\hline $\mathrm{H} 2$ blocker use & 32 & 5 & 5 & 0 & I & 13 \\
\hline Aspiration of gastric contents & 37 & 5 & 7 & I & 0 & II \\
\hline Chronic pain & 5 & 5 & I & I & 0 & 17 \\
\hline Chronic opioid use & 21 & 5 & 4 & I & 0 & 14 \\
\hline Smoking & 68 & 47 & 7 & 3 & 6 & 3 \\
\hline Chronic alcohol use & 10 & 5 & I & 0 & I & 17 \\
\hline Obstructive lung disease & 31.6 & 21.1 & 6 & 4 & 0 & 9 \\
\hline Immunocompromised & 10.5 & 15.7 & l & 2 & I & 15 \\
\hline Diabetes mellitus & 31.6 & 15.7 & 5 & 2 & I & II \\
\hline Body mass index $(B M I) \geq 30$ & 31.6 & 21 & 4 & 2 & 2 & 11 \\
\hline Transfusion & 26 & 5 & 5 & $\mathrm{I}$ & 0 & 13 \\
\hline
\end{tabular}

+/-= matched pair with Recurrent exposed, Single unexposed; $-/+=$ Recurrent unexposed, Single exposed; $+/+=$ both Recurrent and Single exposed; $-/-=$ both Recurrent and Single unexposed.

Table I 4 Association with recurrent $\mathrm{ALI}$ and $\mathrm{No} \mathrm{ALI}^{5}$

\begin{tabular}{|c|c|c|c|c|c|c|}
\hline \multirow[b]{2}{*}{ Variables } & \multicolumn{2}{|c|}{ Exposure frequency } & \multicolumn{2}{|c|}{ Discordant pairs* } & \multicolumn{2}{|c|}{ Concordant pairs* } \\
\hline & Recurrent ALI\% & Single ALI\% & $+1-$ & ++ & $+/+$ & + \\
\hline Gastro-esophageal reflex disease & 79 & 42 & 7 & 0 & 8 & 4 \\
\hline Proton pump inhibitors use & 42 & 42 & 6 & 6 & 2 & 5 \\
\hline $\mathrm{H} 2$ blocker use & 32 & 5 & 5 & 0 & I & 13 \\
\hline Aspiration of gastric contents & 37 & 5 & 7 & l & 0 & II \\
\hline Chronic pain & 5 & 5 & I & I & 0 & 17 \\
\hline Chronic opioid use & 21 & 5 & 4 & 1 & 0 & 14 \\
\hline Smoking & 68 & 47 & 7 & 3 & 6 & 3 \\
\hline Chronic alcohol use & 10 & 5 & I & 0 & 1 & 17 \\
\hline Obstructive lung disease & 31.6 & 21.1 & 6 & 4 & 0 & 9 \\
\hline Immunocompromised & 10.5 & 15.7 & I & 2 & 1 & 15 \\
\hline Diabetes mellitus & 31.6 & 15.7 & 5 & 2 & 1 & II \\
\hline Body mass index $(\mathrm{BMI}) \geq 30$ & 31.6 & 21 & 4 & 2 & 2 & II \\
\hline Transfusion & 26 & 5 & 5 & I & 0 & 13 \\
\hline
\end{tabular}

$+/-=$ matched pair with Recurrent exposed, No ALI unexposed; -/+ = Recurrent unexposed, No ALI exposed; +/+ = both Recurrent and No ALI exposed; $-/-=$ both Recurrent and No ALI unexposed. 
Table I 5 Signs and Symptoms That Suggest Specific Causes of Acute Respiratory Distress Syndrome ${ }^{24}$

\begin{tabular}{|c|c|}
\hline Signs/Symptoms & Possible cause \\
\hline \multicolumn{2}{|l|}{ Most common } \\
\hline Productive cough, fever, pleuritic chest pain. & Pneumonia \\
\hline $\begin{array}{l}\text { Infection plus some of the following findings: temperature }>38.3^{\circ} \mathrm{C} \text { or }<36^{\circ} \mathrm{C} \text {; pulse }>90 \text { beats/minute; } \\
\text { tachypnea; altered mental status; white blood cell count }>12,000 / \mathrm{mm} 3(12 \times 109 \text { per } \mathrm{L}),<4,000 \mathrm{~mm} 3 \\
(4 \times 109 \text { per L), or }>10 \% \text { immature forms; elevated C-reactive protein level; arterial hypotension; acute } \\
\text { oliguria; hyperlactatemia. }\end{array}$ & Sepsis \\
\hline \multicolumn{2}{|l|}{ Less common } \\
\hline History of institutionalization or mental retardation, decreased Glasgow Coma Scale score. & Aspiration \\
\hline History of drug abuse, especially inhalational. & Drug toxicity \\
\hline $\begin{array}{l}\text { Facial burns, singed eyebrows, carbonaceous sputum, and history of working near organic solvents or } \\
\text { toxic chemicals. }\end{array}$ & Inhalation injury \\
\hline History of needing water rescue, hypothermia. & Near drowning \\
\hline Fever, rhinorrhea, cough, history of prematurity or congenital heart disease. & Respiratory syncytial virus \\
\hline Symptoms of acute lung injury and blood transfusion within the previous six hours. & Transfusion-related ALI \\
\hline Bruising over the chest wall, associated injuries, history of motor vehicle crash or fall from a height. & Trauma \\
\hline
\end{tabular}

Table 16 Differential Diagnosis of Acute Hypoxia ${ }^{24}$

\begin{tabular}{|c|c|}
\hline Condition & Clues to Diagnosis \\
\hline \multicolumn{2}{|l|}{ More common } \\
\hline Asthma & Cough, wheeze, response to bronchodilator. \\
\hline Chronic obstructive pulmonary disease & Decreased air movement, prolonged expiratory phase. \\
\hline Congestive heart failure & Jugular venous distension, peripheral edema, third heart sound. \\
\hline Pneumonia* & Productive cough, fever, pleuritic chest pain. \\
\hline \multicolumn{2}{|l|}{ Less common } \\
\hline Acute eosinophilic pneumonia & Fever, cough, diffuse infiltrates, increased eosinophils on bronchoalveolar lavage. \\
\hline Hypersensitivity pneumonitis & $\begin{array}{l}\text { Acute onset; exposure to inciting organic antigen, such as those found in bird feathers, molds, } \\
\text { and dust. }\end{array}$ \\
\hline Pneumothorax & Acute onset of dyspnea, pleuritic chest pain; tall and thin body habitus. \\
\hline Salicylate toxicity & History of suicide attempt, hyperventilation, tachycardia, seizure. \\
\hline Sepsis* & Fever, tachypnea, tachycardia, elevated or depressed white blood cell count. \\
\hline
\end{tabular}

*Pneumonia and sepsis are leading causes of acute respiratory distress syndrome, but may be present in patients who do not meet diagnostic criteria for the syndrome.

Table 17 Factors That Distinguish ARDS, Congestive heart failure and Pneumonia. [+= present, $-=$ not present, $+/-=$ may or may not be present] ${ }^{24}$

\begin{tabular}{|c|c|c|c|}
\hline Distinguishing factor & ARDS & Congestive Heart failure & pneumonia \\
\hline \multicolumn{4}{|l|}{ Symptoms } \\
\hline Dyspnea & + & + & + \\
\hline Hypoxia & + & + & + \\
\hline Tachypnea & + & + & + \\
\hline Pleuritic chest pain & $+/-$ & - & + \\
\hline Sputum production & $+/-$ & - & + \\
\hline \multicolumn{4}{|l|}{ Signs } \\
\hline Rales & + & + & + \\
\hline Fever & $+/-$ & - & + \\
\hline Edema & - & + & - \\
\hline Jugular venous distension & - & + & - \\
\hline Third heart sound & - & + & - \\
\hline \multicolumn{4}{|l|}{ Studies } \\
\hline Hypoxemia & + & + & + \\
\hline Bilateral infiltrates & + & $+/-$ & $+/-$ \\
\hline Pulmonary wedge pressure $\leq 18 \mathrm{mmHg}$ & + & - & - \\
\hline $\mathrm{PaO}_{2} / \mathrm{FiO}_{2} \leq 200$ & + & - & - \\
\hline Localized infiltrate & - & - & + \\
\hline Elevated brain natriuretic peptide level & - & + & - \\
\hline Cardiac enlargement & - & + & - \\
\hline \multicolumn{4}{|l|}{ Responses } \\
\hline Antibiotics & - & - & + \\
\hline Diuretics & - & + & - \\
\hline Oxygen & - & + & + \\
\hline
\end{tabular}


Recent studies indicate that the incidence of ALI/ARDS is 2286 cases per 100,000 person-years and up to 64 cases per 100,000 person-years, respectively. ${ }^{14} \mathrm{~A}$ large, prospective European trial estimated that $7.1 \%$ of patients admitted to an ICU and $16.1 \%$ of all patients on mechanical ventilation develop ALI or ARDS. The inhospital mortality rate for these conditions is estimated to be between 34 and 55\%. Risk factors for mortality include increasing age, worsening multiorgan dysfunction, and presenc of pulmonary and non pulmonary comorbidities, higher Acute Physiology and Chronic Health Evaluation (APACHE) II score, and acidosis. Most ARDSrelated deaths are due to multiorgan failure. Refractory hypoxemia accounts for only $16 \%$ of ARDS-related deaths. In children, ARDS is less common and less likely to lead to death. In a 2009 study of patients six months to 15 years of age, the reported incidences of ALI/ ARDS were 9.5 and 12.8 per 100,000 person-years, respectively, with a combined in-hospital mortality rate of $18 \%$.

However, because the presenting symptoms of ARDS are nonspecific, physicians must consider other respiratory, cardiac, infectious, and toxic etiologies (Table 16). Patient history (e.g., comorbidities, exposures, medications) in conjunction with a physical examination focusing on the respiratory and cardiovascular systems can help narrow the differential diagnosis and determine the optimal course of treatment. Mostly ARDS must be differentiated from congestive heart failure and pneumonia (Table 17). Congestive heart failure is characterized by fluid overload, whereas patients diagnosed with ARDS, by definition, do not show signs of left atrial hypertension or overt volume overload. Patients with congestive heart failure may have edema, jugular venous distension, third heart sound, an elevated brain natriuretic peptide level, and a salutary response to diuretics. Patients with ARDS would not be expected to have these findings. In other aspect, because pneumonia is a leading cause of ARDS, distinguishing patients with uncomplicated pneumonia from those who have pneumonia complicated by ARDS presents a greater diagnostic challenge. In general, a patient with uncomplicated pneumonia may have signs of systemic and pulmonary inflammation (i.e., fever, chills, fatigue, sputum production, pleuritic chest pain, and localized or multifocal infiltrates); accompanying hypoxia should respond to oxygen administration. If hypoxia does not correct with oxygen administration, ARDS should be suspected and confirmed based on AECC diagnostic criteria. In those with combined pneumonia and ARDS, treatment entails antibiotics and ventilator management ${ }^{21}$ (Table 16).

It had been found that a significant number of patients with ALI did not receive care in an ICU; when patients outside the ICU were included, the chance of developing ALI/ARDS with a given clinical insult was substantially lower than reported previously; and the time course from clinical insult to admission to the ICU and diagnosis of ALI/ARDS is rapid, but this process may take longer for extra pulmonary ALI/ARDS. It had been also found that more than half the patients with ALI $\left(\mathrm{PaO}_{2} / \mathrm{FiO}_{2} 200\right.$ to $\left.300 \mathrm{mmHg}\right)$ were managed entirely outside the ICU. This has important implications for the accurate estimation of the true burden of disease in the population, but it also has meaning for clinicians:

a. For clinicians managing patients on medical and surgical wards, it is important to realize that many patients with acute lung injury will be managed entirely outside the ICU. These patients with ALI will need different therapy from patients with cardiogenic pulmonary edema, for whom they may be mistaken.

b. For the intensivist, the question is whether these patients with ALI should be left on the ward, or whether their outcomes would be better if they received care in the ICU (Table 17).
Nonetheless, the death rates were not statistically different between patients with ALI who were admitted to the ICU and those who were not (22\% versus $27 \%)$. Although the confidence intervals around these estimates are wide, they certainly do not suggest that these patients with ALI were kept on the floor because they were all going to do well. It is unknown whether admission to the ICU to receive therapies such as more vigorous resuscitation or non-invasive ventilation would change this outcome (Figure 4). It was found that on average, patients progressed quickly from development of clinical insult, to ICU admission, to diagnosis of ALI/ARDS. The finding that most cases of ARDS occur quickly after the onset of the clinical predisposition is not new; however, the knowledge was extended in two ways:

1. It was found that most patients entering the ICU do so on the day of developing their clinical insult is new, and it underscores the potential need for rapid intervention in these patients.

2. It was observed a significantly longer time to ALI/ARDS development for extrapulmonary risk conditions compared with pulmonary risk conditions (Figure 5).

3. It is worth noting that relatively few patients initially diagnosed with ALI went on to develop ARDS (13\%); this is a significantly lower proportion than the $55 \%$ conversion rate reported in a recent multicentre observational study ${ }^{22}$ (Figure 4).

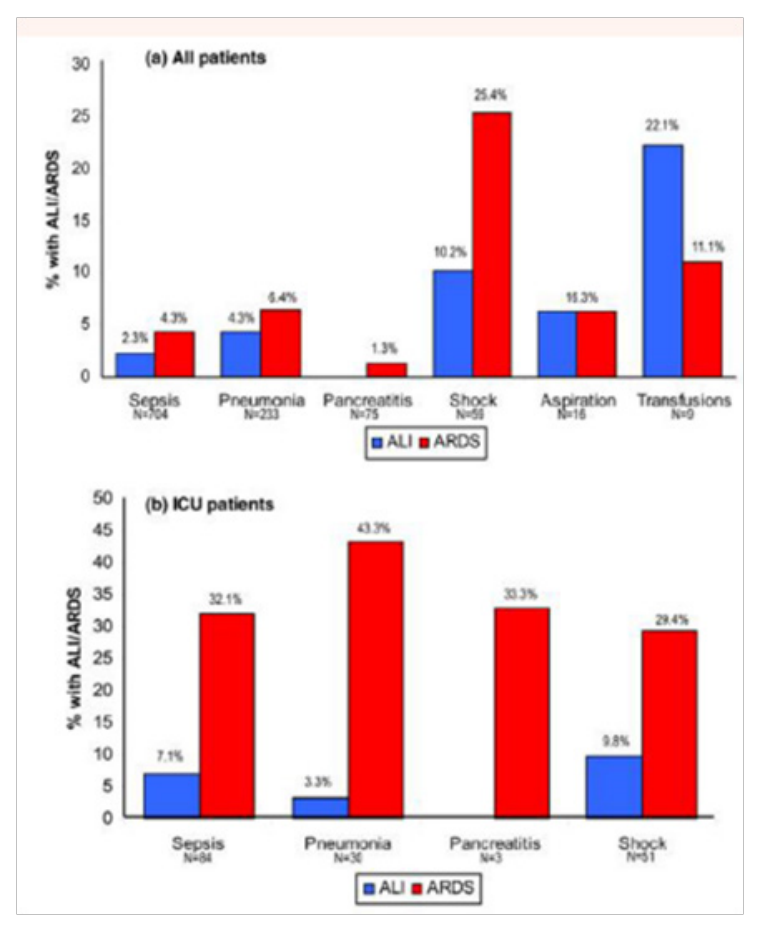

Figure 4 Prevalence of ALI and ARDS by clinical risk condition.

A. The proportion of patients with each clinical risk condition who went on to develop acute lung injury (ALI; blue columns) or acute respiratory distress syndrome (ARDS; red columns) is shown for all patients.

B. And for only those admitted to the intensive care unit.

In both panels the number of patients at risk with each clinical insult is displayed numerically below each category label.

In general it was observed that the time course from clinical insult to diagnosis of lung injury was rapid, but it was longer for extra pulmonary cases. The risk of ARDS was significantly lower than reported previously when patients outside the ICU were considered, but rates in ICU patients appeared similar. A significant number of 
patients with ALI received care outside the ICU, the state of many controversies and laid for many challenge and discussion ${ }^{23}$ (Figure 5).

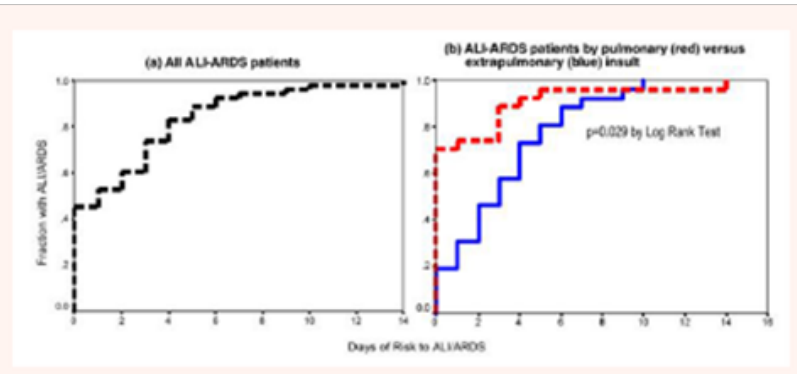

Figure 5 Time from clinical risk to diagnosis of ALI/ARDS.

A. Kaplan-Meier curves displaying time from clinical risk condition to diagnosis of ALI/ARDS are shown for all patients.

B. And separated according to pulmonary (red line) versus extra pulmonary (blue line) risk conditions.

Timing of the onset of acute lung injury and acute respiratory distress syndrome

It was revealed that a new and interesting facts in intensive care medicine regarding the timing of ARDS and its relation to healthcaresystem exposure. It is suggested that ARDS is mainly a healthcarerelated syndrome, as only $14 \%$ of population did not have known healthcare contact prior to ALI/ARDS onset. It is also shown that there is a substantial time gap between hospital admission and ALI/ ARDS onset, which suggests a potential window for future ARDS mechanistic studies and clinical trials of preventive strategies (Figure 6). The principal barrier toward better understanding of the clinical pathogenesis of ALI/ARDS and the design and conduct of ALI/ ARDS preventive strategies is the fact that previous clinical studies focused almost exclusively on patients admitted to the ICU. It is suggested that a substantial time gap is present between the initial development of ARDS risk factors during hospitalization, or even the initiation of mechanical ventilation in critically ill patients admitted to the ICU, and the development of ARDS (Figure 7). It is believed that understanding the time course of ALI/ARDS is the first step toward prevention. Subsequent steps can include developing accurate prediction models to identify patients at high risk of ALI/ARDS, and applying preventive or therapeutic strategies, analogous to other several important critical care syndromes, such as stress ulcer bleed, ventilator-associated pneumonia, central venous catheter infection, and venous thrombo embolism. However, observing the evolution of ARDS in rat lung exposed to injurious ventilation prompted the clinical investigation of the relationship between initial ventilator settings and the development of ARDS in patients who did not have ARDS at the outset. Intriguing preliminary results suggested that the processes of care, namely, high-tidal volume mechanical ventilation and liberal transfusion, were more important ARDS risk factors than the initial severity of illness. ${ }^{24}$ Both ventilator settings and transfusion factors were associated with the development of ARDS, in a dosedependent manner. Indeed, a quality-improvement intervention aimed at decreasing ventilator tidal volume and liberal transfusion decreased harmful exposures and was associated with a reduced incidence of ARDS in mechanically ventilated patients without ALI at the outset (Figure 7).

It was found that patients with community-acquired ARDS were more likely to be medical patients $(85 \%)$ with pneumonia $(59 \%)$, whereas those who developed ARDS after hospital admission were more likely to be surgery patients (54\%). It was suggested that
ARDS due to pulmonary etiologies had worse oxygenation than ARDS due to extra pulmonary causes, which may explain the $\mathrm{PaO}_{2} /$ $\mathrm{FiO}_{2}$ difference between the two types of insult patients, given the fact that the community-acquired ARDS had worse oxygenation and more pneumonia. However, other facts related to possible delayed recognition and treatment of ARDS in the community-acquired group offer an alternative explanation. Although the hospital-acquired ARDS group had lower unadjusted hospital mortality, the difference was not statistically significant after adjusting for baseline differences, which may be due to the small sample size; however, prior studies did not find a mortality difference when comparing pulmonary (more common in the community-acquired ARDS patients) versus extra pulmonary ARDS etiologies. ${ }^{25}$

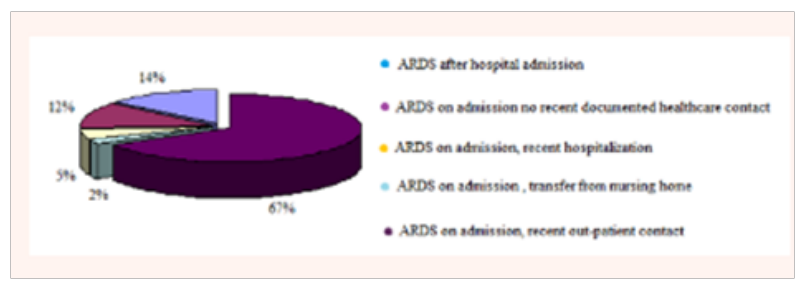

Figure 6 Distribution of patients with acute respiratory distress syndrome in relation to recent healthcare contact.

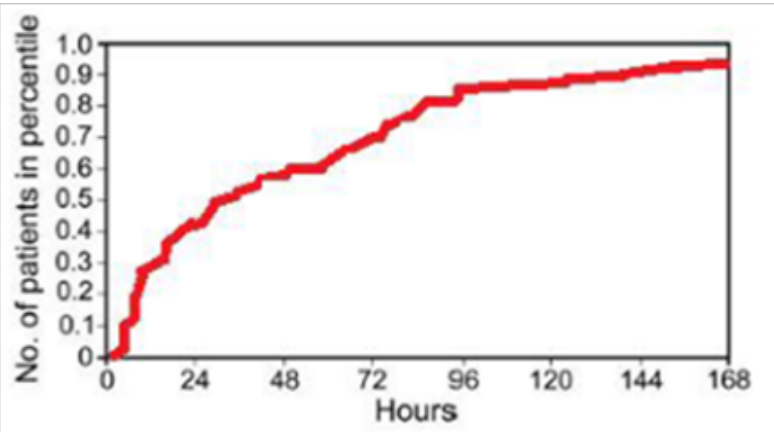

Figure 7 Time to acute respiratory distress syndrome during the first week after hospital admission.

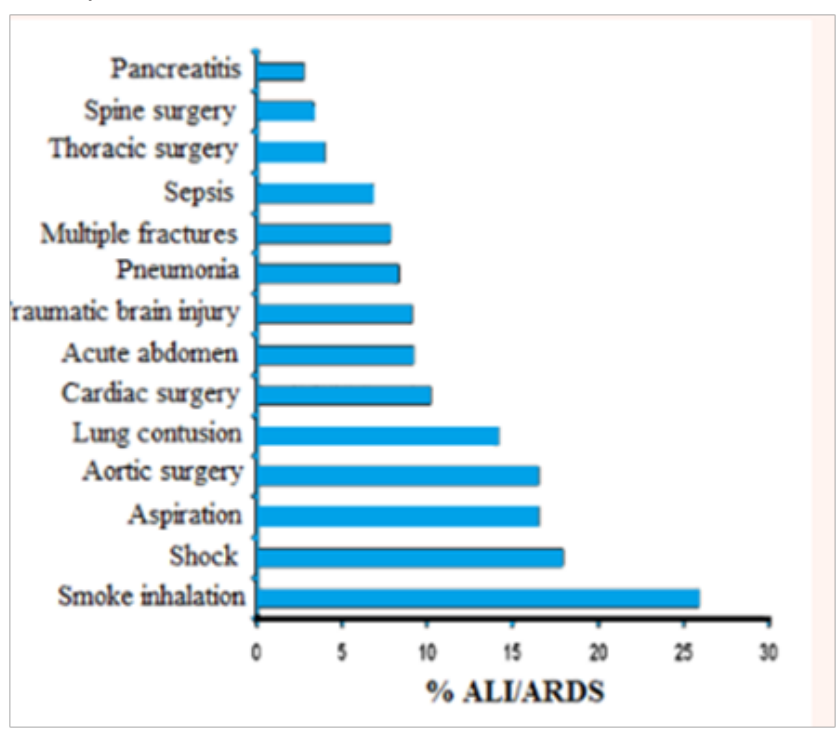

Figure 8 Frequency of ALI/ARDS development according to predisposing conditions. The figure depicts the frequency of ALI/ARDS development in subsets of patients with different risk factors. Because the risk factors are not mutually exclusive, the patients who presented with more than one risk factor may be counted more than once. 


\section{Lung Injury Prediction Score}

Lung injury prediction score [LIPS]: Investigations of therapeutic interventions in acute lung injury and its more severe form, acute respiratory distress syndrome, have concentrated on patients with established disease. Proven and effective treatments at that point are limited. Indeed many treatments targeting the mechanisms identified in promising preclinical studies have failed to improve patient outcomes. Failed trials likely result, in part, from delayed recognition of patients at risk and the subsequent development of the full-blown syndrome. Preventing the development of ALI may be more effective in improving outcomes. Unfortunately, delivering preventative ALI therapies to at-risk individuals has received little attention, in time any intervention decreasing the incidence of ALI will significantly impact the mortality, morbidity, and intensive care unit use associated with this syndrome.

A major obstacle to any early intervention or preventive studies is our inability to anticipate which patients are likely to develop ALI. Epidemiologic data suggest that ALI is rarely present at the time of initial emergency department evaluation or hospital admission for high-risk elective surgery, but develops over a period of hours to days in a subset of at risk patients. A recent Spanish study reported that the vast majority of patients with predisposing conditions never develop ALI, making the enrollment of unselected patients into ALI prevention studies neither feasible nor efficient. Moreover, a large number of patients who ultimately would not develop ALI would be subjected to the risk and expense of a prevention strategy. Recent studies have identified several risk modifiers that may alter the likelihood of ALI development in patients with predisposing conditions. These include alcohol abuse, hypoalbuminemia, tachypnea, oxygen supplementation, chemotherapy, obesity and diabetes mellitus, although whether these factors are independent of one another is unclear. The lack of a validated risk model that confirms and consolidates these risk modifiers prevents the systematic determination of a population at high risk for developing ALI and is a major limitation to studies aimed at prevention or early intervention in ALI.

However, recently observations reported an ALI prediction model, the Lung Injury Prediction Score (LIPS), incorporating the risk factors and risk modifiers present at the time of hospital admission, before ALI onset. It is found that the frequency of ALI varied according to predisposing condition (Figure 8), with the highest rate of ALI occurring after smoke inhalation (26\%) and the lowest rate occurring in pancreatitis (3\%) (Figure 8). The LIPS model has several strengths and it is both unique and easy to perform:

a. It includes clinical information strongly associated with ALI in multiple studies and readily available at the time of the admission. It uses information that is clearly defined and routinely available in the medical record and as part of usual care.

b. The model identifies at-risk patients early in the course of illness and before ICU admission.

c. The model also includes a previously understudied group of patients at high risk for ALI who undergoes high-risk elective (cardiothoracic) surgery.

In clinical practice the LIPS model may potentially be used to alert the providers to patients at risk for ALI. Although no specific intervention has been shown to prevent ALI in patients at risk, applying a model such as LIPS to identify high-risk patients may alert physicians to avoid specific second-hit hospital exposures, such as blood product transfusions, amiodarone, high tidal volume mechanical ventilation, and aspiration. In fact, single-center studies have shown a significant decrease in the incidence of ALI in association with changes in health care delivery. Given the high mortality associated with the development of ALI and the significant functional and cognitive impairment experienced by survivors of ALI, prevention of ALI may improve survival and long-term functional outcomes better than interventions aimed at reducing mortality after development of ALI. Although ALI development markedly increased the risk of death, the observed mortality rate of $23 \%$ is lower than previously reported. The exclusion of patients with established ALI transferred from outside facilities, the inclusion of patients who did not require invasive mechanical ventilation, and secular trends in ALI prognosis provide potential explanations for the observed findings. ${ }^{26}$

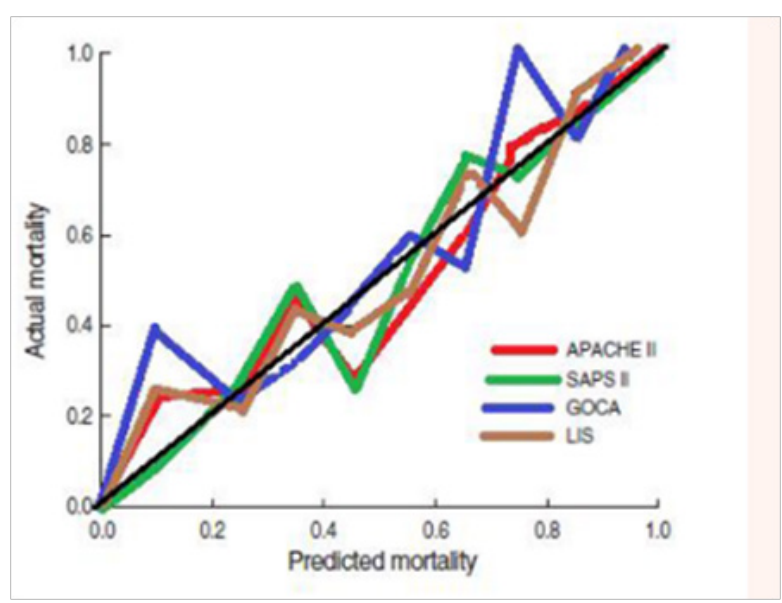

Figure 9 Calibration curves for APAHCE II,SAPS II, GOCA, and LIS. Unmarked line (black line) represents the line of perfect correspondence between actual and predicted risk of death; marked lines (colored lines) represent the calibration curves. The models used for calibration curves were made using patient location before MICU admission in addition to severity scores (Jegal 2008 with modification).

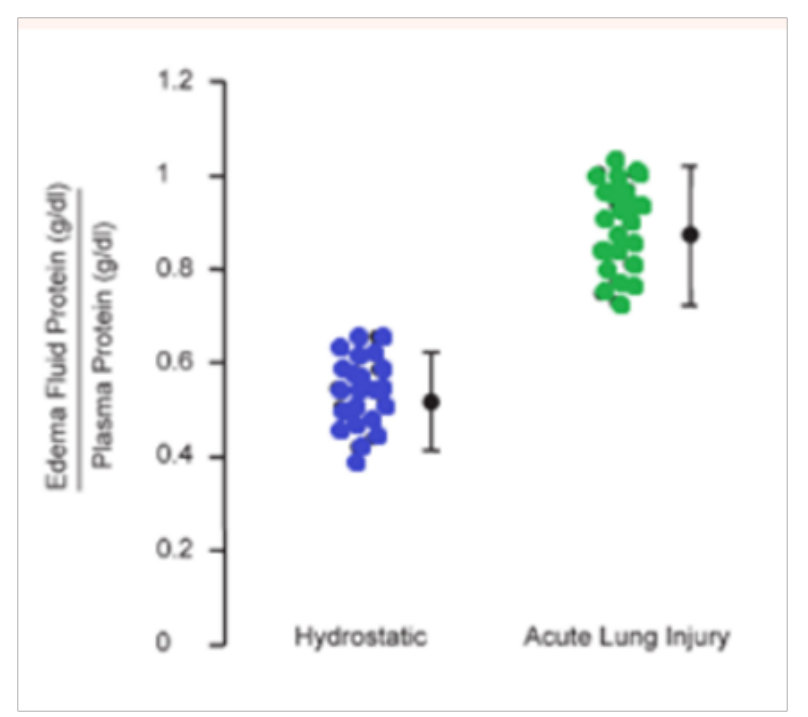

Figure 10 Increased permeability pulmonary edema is a hallmark of ALI/ ARDS. Edema fluid to plasma protein concentrations expressed as a ratio to determine whether the edema fluid is a transudate as in hydrostatic pulmonary edema $(<0.65)$ or an exudate $(>0.65)$ as in increased permeability pulmonary edema (ALI/ARDS). The pulmonary edema fluid samples were obtained from patients within the 15 minutes of entdotracheal intubation for acute respiratory failure. 
The efficacy of GOCA scoring system in patients with ARDS: To compare mortality rates between different institutions, the effects of newer treatment methods, and to identify high mortality groups, a scoring system that can objectively measure the severity-of-illness of ARDS patients would be helpful. Over the past 20 years, there have been numerous efforts to develop models that provide severityof-illness measures to objectively describe ICU populations and ICU risk prediction models were constructed for heterogeneous patients in ICUs. Severity scoring systems that can be applied to patients with ARDS can be divided into two types. One type aims to estimate general health condition of critically ill patients, and the other focuses on the severity of lung injury. Among the severity scoring systems that have been proposed for critically ill patients, the acute physiology and chronic health evaluation (APACHE) and the simplified acute physiology score (SAPS) systems are frequently used. The performances of these systems in ICUs have been reported as satisfactory. APACHE II and SAPS II were originally developed for predicting the prognosis of critically ill patients. Both of these scoring systems focus on evaluating the general health of the patients. By contrast, the lung injury score (LIS) and the Gas exchange, Organ failure, Cause, Associated disease (GOCA) score were developed specifically for patients with ARDS to define or stratify the disease (but not to predict prognosis). LIS mainly focuses on the severity of lung injury. The scoring systems for general critically ill patients may not accurately predict the prognosis in highly specialized patients such as ARDS. Furthermore, the scoring systems that focus on mainly lung condition also can not predict the prognosis of ARDS because the degree of hypoxia was not correlated with the mortality of those patients. Theoretically, GOCA can be superior to APACHE II or SAPS II for predicting the prognosis of patients with ARDS because it considers the severity of lung injury as well as general health condition. The mortality predictive performance of the GOCA system for ARDS patients has not been adequately addressed, and there are few reports that have evaluated the overall performances of LIS, APACHE II, and SAPS II on ARDS patients. However, two hypotheses were formed:

a. One is that neither the severity scoring systems that reflect general condition nor those reflect severity of lung injury can accurately predict prognosis of patients with ARDS when used alone. Therefore, GOCA, which reflects general health condition in addition to the severity of lung injury, can be a better mortality predictor than APACHE II, SAPS II and LIS.

b. The other is that the performance of APACHE II and SAPS II will be improved when they are reinforced by a scoring system that focuses on the severity of lung injury (e.g., LIS) (Figure 9).

The general severity scoring systems, such as APACHE II and SAPS II, showed better performance in predicting mortality of patients with ARDS than LIS, which focuses only on the severity of respiratory failure. APACHE II and SAPS II scores have been previously reported to be independent predictors of hospital mortality in patients with ARDS. However, these studies evaluated the significance of severity scores using only Student t-tests or logistic regression. Although these tests have shown excellent performances on overall critically ill patients, there are only a few reports that have validated the overall performance of these severity scoring systems in ARDS patients. The discriminative powers of APACHE II and SAPS II were considered excellent in previous reports addressing overall critically ill patients. The discriminative powers of these scores were inferior in this study compared to previous studies, possibly because these scoring systems were originally developed for overall critically ill patients, not specifically for patients with ARDS. It was found that LIS was inferior to APACHE II and SAPS II in predicting ARDS mortality. LIS is obtained by dividing the aggregate sum by the number of components that are used. There are four components; the extent of alveolar consolidation (chest radiography) (0-4), the degree of hypoxia (0-4), the degree of respiratory system compliance (0-4), and the degree of PEEP (0-4) (Table 5). The $\mathrm{PaO}_{2} / \mathrm{FiO}_{2}$ ratio was not different between survivors and non-survivors, and this lack of relationship between the degree of hypoxia and mortality might have contributed to the shortage of discriminative power of LIS. The relationship between hypoxia and mortality of ARDS patients remains controversial, as has been reported in previous studies.

Previous studies have also reported that LIS was not suitable for predicting ARDS patient outcome and the discriminative power of LIS for overall survival was inferior to SAPS or APACHE II scores. There are two possible explanations for the inferiority of the LIS:

1. The development of many strategies that support respiratory ability have led to general health condition, rather than lung function, being the major prognostic factor even in patients with ARDS.

2. LIS was originally developed to define ARDS, not to predict prognosis. $^{5}$

The GOCA stratification system was proposed by the American European Consensus Conference on ARDS to assess the severity of acute lung injury and the associated clinical features. GOCA considers not only the severity of lung injury but also a few general conditions such as the number of organ failure and associated diseases. The GOCA score is the sum of four variables:

A. The severity of gas exchange (0-3).

B. The number of organ failure (0-3).

C. The cause of lung injury ( 0 : lung only, 1 : direct lung injury, and 2: indirect lung injury)

D. The associated diseases ( 0 : no associated disease that will cause death within 5 years, (coexisting diseases that will cause death within 5 years but not within 6 months, and 2: coexisting diseases that will cause death within 6 months).

Although the GOCA score is not designed to predict outcome, it have hypothesized that GOCA can be better than the other scoring systems in predicting prognosis of ARDS patients because it considers the general health condition of patients in addition to the severity of lung injury. The results generally did not, however, show any predictive advantage of the GOCA system. This may be because the scoring for associated diseases in GOCA is dependent on the subjective judgment of the attending physician and the differentiation of primary disease is too crude. On the other hand, GOCA may prove to be more convenient to use than APACHE II or SAPS II because it requires fewer variables but provides the same predictive power. ${ }^{27}$

ALI/ARDS prediction score: Acute lung injury and its more severe form acute respiratory distress syndrome are examples of critical care syndromes with limited treatment options once the condition is fully established. Pre-clinical studies support a two-hit model of ALI/ARDS development whereby exposure to pertinent risk factors modify the development and expression of ALI/ARDS in an already susceptible host with predisposing conditions. The condition usually develops in patients with underlying risk factors (pneumonia, severe sepsis, trauma and aspiration) but is modified by different patient's 
characteristics including genetic predisposition, as well as certain medical interventions (adverse ventilator settings and transfusion of alloimunised plasma). Animal models provide compelling evidence in support of oxidative stress, lung deformation, loss of compartmentalization of inflammation and intravascular coagulation as the pathogenic mechanisms involved in the development of ALI/ ARDS. However, many treatments targeting these mechanisms have failed to improve patient outcomes despite compelling preclinical data. It is probable that inadequate or delayed recognition and treatment of patients at risk of the full-blown syndrome have obscured the therapeutic window. The recent National Institute of Health workshop prioritized the development of strategies to perform ALI/ARDS prevention trials. The epidemiological data suggest that ALI/ARDS is rarely present at the time of hospital admission. Rather, ALI/ARDS appears to develop over a period of hours to days in this subset of patients at risk. Unfortunately, clinical studies are usually performed in the ICU setting, enrolling patients with established ALI/ ARDS who are beyond the therapeutic window of potential prevention strategies. This delayed enrolment prevents adequate study of patients at risk.

A significant challenge with early enrolment of patients at risk of ALI/ARDS into prevention trials is the fact that the majorities of patients with predisposing conditions never develops ALI/ARDS and are never admitted to the ICU. This makes the enrolment of unselected patients into ALI/ARDS prevention studies neither feasible nor efficient. The likelihood of ALI development depends not only on specific risk factors (from $5 \%$ with elective cardiopulmonary bypass to $40 \%$ in patients with septic shock), but also on the presence of specific risk modifiers. These include alcohol abuse, smoking, hypoalbuminemia, tachypnea, oxygen supplementation, chemotherapy and diabetes mellitus. When comparing the validation cohort (hospitalized patients regardless of ICU disposition at the time of admission) to the derivation cohort (only ICU patients), the proportion of patients with risk factors who developed ALI was markedly reduced. Similar results were recently published by Ferguson et al where only $7 \%$ of hospitalized patients with sepsis, $2 \%$ with pancreatitis, $10 \%$ of patients with pneumonia and $15 \%$ of patients with witnessed aspiration developed ALI. Indeed, the majorities of patients with predisposing conditions never develops ALI/ARDS and are never admitted to the ICU. ${ }^{28}$ This makes the enrolment of unselected patients into ALI/ARDS prevention studies neither feasible nor efficient without a method for identifying those who are at high risk. The failure to take into account multiple triggers that influence ALI/ARDS development has probably led to the discarding of a number of potentially important therapeutic advances in ARDS which may prove to be effective in specific and highly characterized groups of patients, particularly if applied early in the course of illness. While some previous studies reported the increased risk of ALI/ARDS in the elderly, other studies have not confirmed this association. It could be argued that elderly patients seem to have an increased incidence of ALI/ARDS as they tend to have more sepsis, pneumonia and aspiration, and require more medical interventions. However, in patients admitted to the hospital with a risk factor (pneumonia or sepsis), age does not seem to increase the risk of ALI/ARDS development. Indeed, recent work implies that incidence of ALI/ARDS due to community-acquired pneumonia is lower in patients aged $\geq 85$ years. ${ }^{29}$

\section{ALI/ARDS: Mechanism Overview}

Pulmonary edema and the early events in ALI/ARDS: Congestion, atelectasis, and pulmonary edema were features of the original description of the syndrome. Within a decade, clinical and experimental studies established the concept that increased permeability pulmonary edema is the primary physiologic abnormality in the early stages of ALI/ARDS. Large animal models with measurements of hemodynamics and lung lymph flow demonstrated that clinically relevant causes of ARDS, including live bacteria, endotoxin, and microemboli, induced an increase in lung vascular permeability that causes protein-rich lung edema. An important clinical study reported protein concentrations in the undiluted edema fluid of mechanically ventilated patients with acute respiratory failure from severe pulmonary edema. In all subjects with features consistent with ARDS, the ratio of the protein concentration in the edema fluid compared with than the simultaneously measured plasma sample was $>0.75$, whereas patients with cardiogenic or high pressure, pulmonary edema had an edema fluid to plasma protein ratio $<0.75$. More recent observations are consistent with this finding, showing a separation between patients with hydrostatic versus an increased permeability pulmonary edema (Figure 10). Thus, increased permeability edema, interpreted as accumulation of protein- rich edema fluid into the alveoli, has become a hallmark of ALI/ARDS (Figure 11).

Anyhow, subsequently many studies have been done to define mechanisms that account for the acute increase in lung vascular permeability. Earlier experiments concentrated mostly on large animal models in which pulmonary and systemic hemodynamics could be measured, whereas more recent studies have used mouse models, largely to exploit the opportunity to use specific gene deletions to define molecular events that may be pivotal in the development of altered lung vascular permeability. At now mostly the in vitro models have used cultured endothelial cells. In parallel with early attempts to understand the mechanisms that lead to lung edema factors that compound the physiologic derangements were considered. One hypothesis was that ALI is associated with surfactant dysfunction, either because of reduced production or neutralization of surfactant by the plasma proteins and fibrin that extravasate into the alveoli. A decrease in functional surfactant would contribute to alveolar instability and arterial hypoxemia, potentially increase lung edema formation and perhaps impair innate immune defenses. Analysis of bronchoalveolar lavage samples indicates that the lipid and protein components of surface active material are altered in patients with ALI/ARDS. Nevertheless, replacement of surfactant has not reduced mortality in large clinical trials in adults with ALI, perhaps because, unlike infant respiratory distress syndrome, the fundamental lesion in the acute respiratory distress syndrome is not lack of surfactant production by immature alveolar type II cells. In sharp contrast, surfactant replacement has substantially reduced mortality in infant respiratory distress syndrome in preterm infants. The role of lung epithelial cell injury and dysfunction and the mechanisms that regulate removal of alveolar edema fluid, which were not recognized in early considerations of ALI/ARDS, emerged in experimental and clinical studies in the 1980s and 1990s. The concept of lung fluid balance incorporates both formation and removal and provides a more dynamic concept of the factors that contribute to the net quantity of edema in the lung in animals or humans with ALI (Figure 11). Edema formation and accumulation in the interstitium and airspaces of the lung may be substantial because of a marked increase in lung vascular permeability, but if alveolar epithelial fluid reabsorption balances the formation of alveolar edema, then a steady state can be established that may allow time for recovery from the fundamental cause of lung injury. Perhaps this is why patients who maintain higher rates of alveolar epithelial fluid transport in the face of ALI have a better survival. In addition, although a primary event in ALI/ARDS is an increase in lung vascular permeability, it was apparent from experimental models and clinical observations that the magnitude of lung edema in ALI may be substantially increased when lung vascular pressures and volume 
are elevated, consistent with the effects of hydrostatic pressure on transvascular flux of fluid and protein. More recently, a large clinical trials are now testing the hypothesis that measures to lower lung vascular pressures in patients with ALI/ARDS can improve clinical outcomes by reducing the quantity of extravasated protein-rich edema fluid in the lung, thus reducing the severity of respiratory failure and ultimately decreasing mortality. In addition to these investigations, the intimate relationship between alveolar edema formation and inflammatory and thrombotic effector mechanisms gradually emerged in the decades after the clinical and physiologic presentations of ALI/ ARDS were described. These concepts were integral to the clinical studies that demonstrated the variety of triggering disorders that can be associated with the development of ALI, resulting in injury to the alveolar barrier (Figure 11).

Inflammatory mechanisms in ALI/ARDS: As commonly revealed that the altered alveolar barrier function and pulmonary edema are central characteristics of ALI/ARDS, mechanisms that could account for the increase in permeability of the alveolar-capillary membrane were poorly understood. Even in early years, however, some investigators believed that inflammation might be involved. One stimulus for this view was the presence of leukocytes together with alveolar edema, hemorrhage, and hyaline membranes in some autopsy specimens (Figure 12). Although subsequent pathologic studies were hampered by heterogeneity in predisposing conditions underlying ALI/ARDS (Figure 11), the risk of lung biopsy in respiratory failure, and the timing and relative infrequency of autopsies, they still provided key information. One study reported purulent exudates in the alveoli of some patients, although the contributions of infection versus inflammatory injury were not dissected. The ultrastructural studies of the lung in patients dying with ALI secondary to sepsis were demonstrated an increased numbers of intravascular and extravascular neutrophils (PMNs), platelets, and fibrin, and both endothelial and epithelial injury, findings that are still incorporated into recent concepts of inflammatory edema in ARDS (Figure 12). Additional pivotal observations included the presence of PMNs and other leukocytes in bronchoalveolar lavage samples from subjects with ALI/ARDS, studies of PMN-dependent lung injury and edema in animal models, and evidence in cell biologic and in vivo analyses that PMN oxidants and proteases can injure cells of the alveolar-capillary membrane. Subsequent observations are consistent with the concept that inflammation is a component of many, and perhaps all, causes of direct injury to the alveolar-capillary membrane as occurs with aspiration and many forms of infectious pneumonitis (Figure 11). The recent studies of complement activation, specifically generation of $\mathrm{C} 5 \mathrm{a}$, and consequent activation of PMNs contributed a new facet to concepts of the pathogenesis of ALI/ARDS. Together with reports demonstrating endothelial injury by PMN proteases and oxidants, these observations provided a conceptual model in which generation of a circulating mediator (in this case, C5a) induces systemic activation of PMNs, resulting in aggregation and sequestration in pulmonary microvessels and consequent diffuse lung injury. This suggested a mechanism by which indirect alveolar-capillary membrane injury is caused, as in systemic sepsis and non-thoracic trauma. Leukocyte activation leading to intravascular sequestration, alone or involving interacting platelets is consistent with findings in much more recent studies of the pathophysiology of ALI/ARDS. These concepts of PMN activation and aggregation also contributed to early suggestions that glucocorticoids, inhibitors of PMN granular proteases, and antioxidants might be specific therapeutic agen agents [a potential that, although mechanistically based, has not subsequently been realized] (Figure 13).

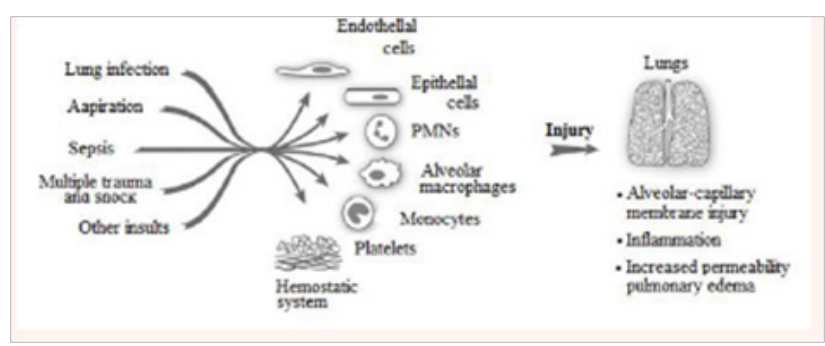

Figure I I Early events in ALI/ARDS. A variety of direct (lung infection, aspiration) and indirect (sepsis, multiple trauma with shock and large volume blood replacement) clinical insults lead to ALI. Initial clinical descriptions identified pulmonary edema as a major consequence. Subsequent investigations yielded evidence for inflammatory injury to the alveolar-capillary membrane as a central pathogenetic mechanism. The key effector cells, molecules, and mechanisms that lead to dysregulation of inflammatory and haemostatic pathways in ALI/ARDS remain incompletely defined.

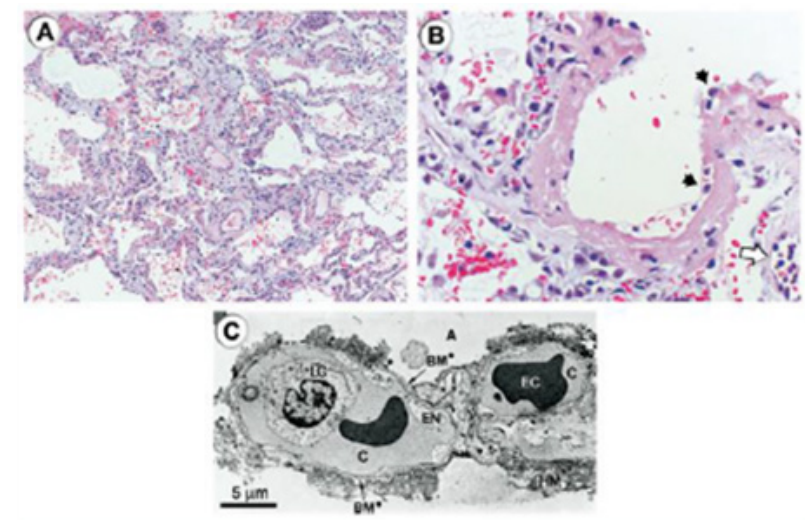

Figure 12 Histologic and ultrastructural analysis of the injured lung has been integral to current concepts of pathogenesis of ALI/ARDS.

A. A low-power light micrograph of a lung biopsy specimen collected 2 days after the onset of ALI/ARDS secondary to gram-negative sepsis demonstrates key features of diffuse alveolar damage, including hyaline membranes, inflammation, intra alveolar red cells and neutrophils, and thickening of the alveolar-capillary membrane.

B. A higher-power view of a different field illustrates a dense hyaline membrane and diffuse alveolar inflammation. Polymorphonuclear leukocytes are imbedded in the proteinaceous hyaline membrane structure (black arrows). The white arrow points to the edge of an adjacent alveolus, which contains myeloid leukocytes.

C. An electron micrograph from a classic analysis of ALI/ARDS showing injury to the capillary endothelium and the alveolar epithelium. LC refers to a leukocyte (a neutrophil) within the capillary lumen. EC designates erythrocytes. EN shows blebbing of the capillary endothelium. BM refers to exposed basement membrane where the epithelium has been denuded. $C$ refers to the capillary. A refers to the alveolar space.

Additional analyses of leukocytes in the blood and alveoli of subjects with ALI/ARDS indicated that accumulation and activation of PMNs could not be completely explained by existing information's and known mediators such as $\mathrm{C} 5 \mathrm{a}$, and stimulated new investigators in the field to develop alternative models and hypotheses and to draw on previous and parallel investigations of inflammatory pathways in other systems. One outcome was identification of endothelial cell activation as a mechanism of leukocyte accumulation and signaling. Thus, key mediators might not be acting directly on the leukocytes but instead on the lung endothelium, resulting in accumulation of PMNs, inflammatory injury, and, potentially, accumulation of other leukocyte types in the injured lung. A key concept in current pathobiology is that endothelial activation is central to inflammation in the lung 
and elsewhere. ${ }^{30}$ Subsequent studies have examined mechanisms that regulate leukocyte-endothelial interactions in the pulmonary versus systemic circulations, where there are both differences and similarities. These issues have additional complexity because ALI/ ARDS can involve both pulmonary and systemic vascular beds.

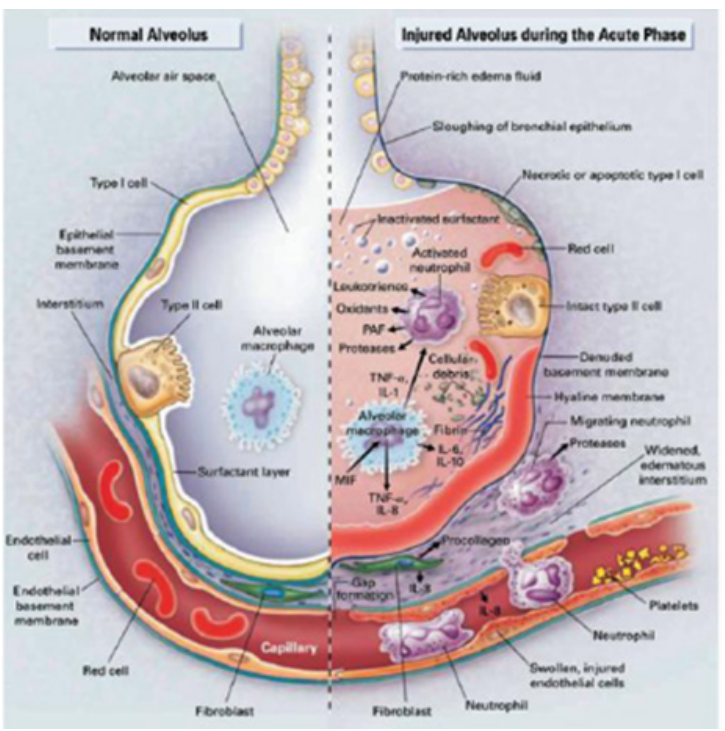

Figure I 3 Multiple cellular responses and mediators contribute to alveolarcapillary membrane injury (right hand side) and the transition from normal alveolar structure and function (left-hand side) in the acute phase of ALI/ ARDS. Original investigations of the pathogenesis of ALI/ARDS searched for single mediators that provided final common pathways to inflammation and alveolar Edema in ALI/ARDS. Current concepts of pathogenesis involve multiple molecular factors of several classes, a variety of responding cells, and imbalance between injurious and reparative signals and pathways. See text for more details.

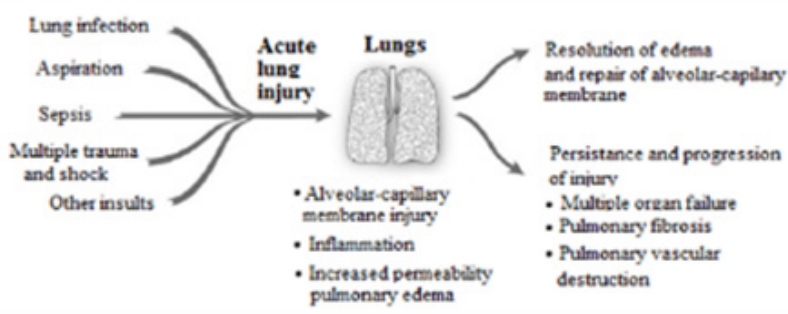

Figure 14 The natural history of ALI/ARDS includes resolution and repair versus persistence and progression. Clinical and epidemiologic studies demonstrate thatALI/ARDS resolves with return of alveolar function to normal or near normal in some patients, whereas in others there is persistence and/ or progression of injury. The outcomes of persistence and progression include multiple organ failure, fibrosing alveolitis, pulmonary vascular obliteration with pulmonary hypertension, and death. The genetic, cellular, molecular and iatrogenic factors that contribute to each of these outcomes remain largely unknown. In addition, rational mechanism-based strategies that favorably influence repair of the alveolar-capillary membrane are undefined.

HMBG-1, and chemokines and also directly interact with PMNs and monocytes in addition to organizing fibrin clots; thus, they are active in innate immune cascades and may modify inflammatory injury in ALI/ARDS. In parallel, however, platelets also release sphingosine-1-phosphate, which promotes endothelial barrier function in experimental models. This illustrates the well known fact that platelets have both defensive and reparative activities. Thus, it is not yet clear if their accumulation and activation in the lungs has a net positive or negative effect on the outcome of ALI/ARDS. Also, in addition the animal and human studies demonstrate that mechanical factors and the pattern of ventilation induce or amplify alveolar inflammation and injury and contribute to nonpulmonary organ injury. Thus, unfavorable ventilation strategies can compound the severity of ALI, adding an iatrogenic variable to classic triggers of injury. Oxygen in high concentrations, which is vital in the support of patients with ALI/ARDS, can also be toxic to alveolar-capillary membrane cells of animals and humans and presents another mechanism of iatrogenic injury, although one recent clinical trial found no decrease in mortality when a lower fraction of inspired oxygen was used in patients with higher levels of positive endexpiratory pressure. In animal models, hyperoxia increases intrapulmonary retention of PMNs and induces dysregulation of other innate immune mechanisms. Inflammatory cytokines can worsen or, conversely, ameliorate oxygen injury in surrogate models (Figure 14). 3 The concept of ALI mediated by PMNs and circulating or locally generated mediators (Figure 13) with origins outlined above, continues to be central to current concepts of the pathogenesis of ALI/ARDS and has been emphasized in reviews and symposia that span two decades. Inflammation was identified as a key feature in the 1994 Consensus conference on ALI/ARDS. The concept has also evolved in complexity and detail. C5a, which remains topical but is not a final common pathway to ALI/ARDS, is now complemented by chemokines, cytokines, and lipid signaling molecules. PMNs are themselves sources of some of these factors. In some cases, identification of individual inflammatory mediators in cell biologic and preclinical models has generated candidate therapeutic strategies that have been tested in clinical trials. Intracellular signaling pathways that link PMN surface receptors to activation responses, including kinases and the NF- $\mathrm{KB}$ family of transcription factors, are implicated as potential points of intervention, and it is recognized that these pathways are modulated by surface integrins, selectins, and selectin ligands, which are adhesion molecules that also mediate tissue accumulation of leukocytes. PMN activities besides acute generation of oxidants and release of proteases, such as signaling of endothelium, inflammatory gene expression and apoptosis are believed to be relevant. There is evidence that injurious versus protective responses of PMNs may influence outcomes and that there are time-dependent changes in PMN number and phenotype in ALI/ARDS, although the significance of these findings remains incompletely explored. It also seems unlikely that PMNs act alone in any of the phases of ALI/ ARDS (Figure 13), although they may have particular roles at acute and subacute time points. Lung macrophages, circulating monocyte subpopulations, and other leukocyte subtypes have been suggested as key effector cells and have been considered in a preliminary fashion in both early and later studies, although little functional data exist. The roles of platelets, which were detected in the acutely injured lung in early studies and molecular and cellular links between the thrombotic and inflammatory systems, remain topical issues. This is in part because of the potential therapeutic use of recombinant activated protein $\mathrm{C}$ (APC) in sepsis, a key triggering conditions for ALI/ARDS. APC interrupts both inflammatory and thrombotic events in experimental studies. Platelets release IL-1

Resolution of lung injury: The natural history of ALI/ARDS has been progressively defined by clinical studies and follows a variable course. One outcome is resolution and repair (Figure 14). The successful resolution of pulmonary edema and lung inflammation is an important determinant of recovery from acute lung injury. Early studies demonstrated that lung lymphatics and the pulmonary microcirculation remove edema fluid from the interstitium of the lung and that fluid flow into the pleural space is an additional major pathway for edema translocation. A new mechanism was recognized in the 1980s when investigators demonstrated that removal of alveolar edema fluid depends on orientation transport of salt and water across 
the alveolar epithelium in part through apical located epithelial sodium channels $(\mathrm{ENaC})$, followed by extrusion into the lung interstitium via a basolaterally located Na-KATPase (Figure 15). The transport of sodium creates a mini- osmotic gradient that absorbs water from the airspaces via water channels (aquaporins) and paracellular pathways. ${ }^{31}$ Net alveolar fluid clearance can be upregulated by cAMP agonists in many species, including the human lung. Recent evidence suggests that the cystic fibrosis chloride channel, CFTR, is required for effective cAMP stimulated fluid transport. Both alveolar type II and type I cells may be capable of driving sodium transport and net alveolar fluid clearance. The transport capacity of the alveolar epithelium is markedly diminished in ALI, a finding that correlates with higher mortality. The mechanisms for the decrease in alveolar fluid clearance include frank injury to alveolar epithelial cells and their apoptosis or necrosis, resulting in loss of barrier and transport properties as well as more subtle defects in ion transport capacity. Oxidant injury, reactive nitrogen species, hypoxia, and direct effects of bacterial and viral pathogens alter the transport machinery in theepithelial cells, as do unfavorable ventilatory strategies (Figure 15).

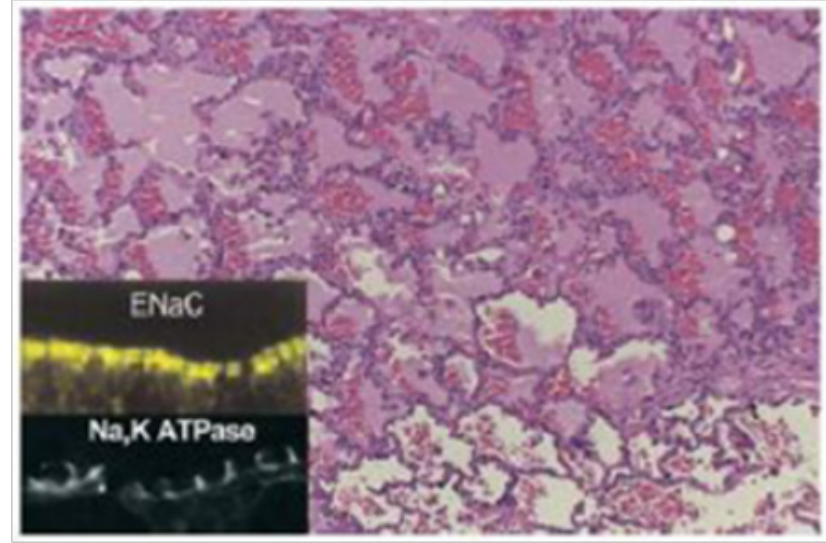

Figure 15 Cellular and molecular pathways regulate the resolution of alveolar edema formation and resolution in ALI/ARDS. Histologic section from patients with a lung biopsy in the setting of lung injury from bacterial pneumonia and sepsis with protein rich alveolar edema. The insert refers to $\mathrm{ENaC}$, the epithelial sodium channel, which is a major pathway for the uptake of sodium on the apical surface of alveolar epithelial type I and II cells as well as distal airway epithelia. NaKATPase refers to the sodium pump located in the basolateral surface of alveolar and airway epithelia that actively pumps sodium into the interstitial space, thus creating a mini-osmotic gradient for the absorption of edema fluid from the alveolar. Both ENaC and NaKATPase can be up regulated by several catecholamine dependent and independent mechanisms.

More precise understanding of how the alveolar endothelium and epithelium interact, and their coordinate responses to inflammatory cells and cytokines, could clarify mechanisms by which lung endothelial injury leads to alveolar edema (Figure 11) and how resolution of edema is modulated (Figures 14 \& 15). Experimentally, alveolar endothelial injury from endotoxin can occur without accumulation of alveolar edema, apparently because epithelial injury is mild. In contrast, substitution of live pathogenic bacteria results in both endothelial and epithelial injury. Since direct or indirect infection accounts for more than $60 \%$ of clinical cases of ALI in most studies, a better understanding of how the bacterial and viral products alter these and other aspects of alveolar-capillary function is critical. Furthermore, it is essential to determine how well the alveolar epithelium recovers from apoptotic or necrotic lung injury and the basic mechanisms that regulate this process of epithelial repair. It is also critical to understand more about how inflammatory cells and soluble and insoluble proteins are removed from the lung. Characterizations of molecular mechanisms that regulate macrophage-dependent removal of apoptotic leukocytes in inflammatory lung injury are in progress. Soluble protein appears to be primarily removed by a slow process of paracellular diffusion across the alveolar epithelium although transcytosis can contribute as well. Removal of insoluble proteins and cellular debris and remodeling of hyaline membranes appears to be primarily driven by alveolar macrophages.

Where we are now: In spite of elements of progress, much remains to be learned. This was apparent to an interdisciplinary group of investigators that met to discuss the state of the field and future research priorities in ALI/ARDS in 2002. The issues are broad and the unknown's complex. However, in all of the facets of the inflammatory and haemostatic responses in ALI/ARDS (Figures $11 \&$, a regulatory cytokine, has important activities that modulate inflammation and edema, but recent evidence suggests that it may also enhance endothelial and epithelial permeability and have a net deleterious effect in the acutely injured lung.(313) we still have little insight into what is cause and what is consequence. Although some progress has been made, we are little closer than we were in 1967 to understanding how key inflammatory events that are defensive and reparative when they are regulated in lung infection and limited injury become damaging and destructive when they are unregulated in ALI/ARDS, and we have very little insight into what the molecular mechanisms of dysregulation actually are. In bacterial pneumonia, innate immune responses mediated by activated neutrophils, monocytes, and alveolar macrophages play requisite roles. In parallel, a local shift to a procoagulant and antifibrinolytic environment may serve to wall off infection and airspace injury, yet these same effectors and responses appear to be essential in the early events in ALI/ARDS (Figure 11). The concept of a balance between pro- and anti-inflammatory and pro- and anti-edematous factors has current traction in the field, in part borrowed from other diseases and mechanisms of injury. Further, specific regulatory molecules may have essential activities that usually keep lung inflammation and edema in check. Nevertheless, while the concepts are objecting, the defining mechanisms remain obscure and many molecular factors may play some effect. As an example, transforming growth factor.

The drive to understand initiation of ALI, the acute inflammatory components of ALI/ARDS, and their resolution (Figure 11) has been accompanied by focused intent to define cellular and molecular events that contribute to progressive alveolar-capillary injury and dysregulated repair (Figure 14), particularly in the last decade of molecular medicine. It is clear from clinical and epidemiologic studies that specific biologic features prevent the resolution of ALI in some subjects, resulting in persistence and progression to severe complications including multiple organ failure, fibrosing alveolitis, and alveolar endothelial and epithelial cell destruction (Figures 12 \& 14). The understanding here is extremely limited. Further progress will likely require more cell biologic experiments and models to establish molecular effects that can then be explored in animal models and, ultimately, in critically ill patients. In addition, genetic determinants of these responses and how they vary among individuals and interact with environmental influences are not largely explored. Cellular, genomic, proteomic, and lipidomic patterns in the acutely injured lung and in lungs undergoing regulated repair versus dysregulated progression remain to be defined. Thus, the biologic basis required for clear understanding of the natural history of ALI/ARDS and for new strategies for intervention remains incompletely defined. Finally, the success of a lung protective ventilatory strategy in reducing mortality in ALI/ARDS is a good example of how clinical trials are needed 
to test novel hypotheses that derive initially from preclinical studies. The marked decrease in mortality with lung protective ventilation has evolved, as outlined earlier in this review, from animal studies that suggested that the standard approach of ventilating patients with ALI/ARDS with higher tidal volumes could amplify the severity of lung injury. Knowledge of the responses of individual cell types to mechanical and inflammatory stimuli provided insight into how this might occur at a molecular level. Subsequently, the success of lung protective ventilatory strategies has stimulated new investigations into how mechanical stresses may adversely affect the lung. The mechanisms of benefit are still being worked out, but seem to involve a modest decrease in lung and systemic inflammation and a reduction in the severity of alveolar epithelial injury. The reduction in mortality with the reduction in tidal volume and plateau airway pressure has been accompanied by a decrease in nonpulmonary organ failure, thus clearly linking the severity of the lung injury to the systemic consequences of ALI/ARDS. ${ }^{32,33}$ However, one of up to date progression in the filed of ALI/ARDS is the Berlin definition.

\section{Acknowledgments}

None.

\section{Funding}

None.

\section{Conflicts of interests}

The authors have no conflicts of interest to declare.

\section{References}

1. Augustine M K Choi. Acute Respiratory Distress Syndrome, Second Edition (Lung Biology in Health and Disease). Informa Healthcare USA. 2010

2. Ashbaugh DG, Bigelow DB, Petty TL. Acute respiratory distress in adults. Lancet. 1967;2(7511):319-323.

3. Raghavendran K, Napolitano LM. ALI and ARDS: Challenges and advances. Crit Care Clin. 2011;27(3):429-437.

4. Sloane PJ, Gee MH, Gottlieb JE, et al. A multicenter registry of patients with acute respiratory distress syndrome. Physiology and outcome. Am Rev Respir Dis. 1992;146(2):419-426.

5. Murray JF, Matthay MA, Luce JM, et al. An expanded definition of the adult respiratory distress syndrome. Am Rev Respir Dis. 1988;138(3):720-723.

6. Atabai K, Matthay MA. The pulmonary physician in critical care c 5: Acute lung injury and the acute respiratory distress syndrome: definitions and epidemiology. Thorax. 2002;57(5):452-458.

7. Villar J, Perez-Mendez L, Lopez J, et al. An early PEEP/FIO2 trial identifies different degrees of lung injury in patients with acute respiratory distress syndrome. Am J Respir Crit Care Med. 2007;176(8):795-804.

8. Britos M, Smoot E, Liu KD, et al. The value of PEEP and $\mathrm{FiO}_{2}$ criteria in the definition the acute respiratory distress syndrome. Crit Care Med. 2011;39(9):2025-2030.

9. Moss M, Goodman PL, Heinig M, et al. Establishing the relative accuracy of three new definitions of the adult respiratory distress syndrome. Crit Care Med. 1995;23(10):1629-1637.

10. Goh AYT, Chan PWK, Lum LCS, et al. Incidence of acute respiratory distress syndrome: a comparison of two definitions. Arch Dis Child. 1998;79(3):256-259.

11. Villar J, Kacmarek RM. The American-European Consensus Conference definition of the acute respiratory distress syndrome is dead, long live positive end-expiratory pressure! Med Intensiva. 2012;36(8):571-575.
12. Ranieri VM, Rubenfeld GD, Thompson BT, et al. Acute respiratory distress syndrome: the Berlin definition. ARDS Definition Task Force. JAMA. 2012;307(23):2526-2733.

13. Pinheiro BV, Muraoka FS, Assis RV, et al. Accuracy of clinical diagnosis of acute respiratory distress syndrome in comparison with autopsy findings. J Bras Pneumol. 2007;33(4):423-428.

14. Rubenfeld GD, Caldwell E, Peabody E, et al. Incidence and outcomes of acute lung injury. New Engl J Med. 2005;353(16):1685-1693.

15. Terashima T, Matsubara M, Nakamura M, et al. Local Pseudomonas instillation induces contralateral injury and plasma cytokines. $\mathrm{Am} J$ Respir Crit Care Med. 1996;153(5):1600-1605.

16. Goodman LR, Fumagalli R, Tagliabue P, et al. Adult respiratory distress syndrome due to pulmonary and extrapulmonary causes: CT, clinical, and functional correlation. Radiology. 1999;213(2):545-552.

17. Pelosi P, D'Onofrio D, Chiumello D, et al. Pulmonary and extrapulmonary acute respiratory distress syndrome are different. Eur Respir J. 2003;42:48s-56s.

18. Bakowitz M, Bruns B, McCunn M. Acute lung injury and the acute respiratory distress syndrome in the injured patient. Scand J Trauma Resusc Emerg Med. 2012;20: 54.

19. Heffernan DS, Dossett LA, Lightfoot MA, et al. Gender and ARDS in critically injured adults: a prospective study. J Trauma. 2011;71(4):878885 .

20. Bice T, Li G, Malinchoc M, et al. Incidence and risk factors of recurrent Acute Lung Injury. Crit Care Med. 2011;39(5): 1069-1073.

21. Saguil A, Fargo M. Acute respiratory distress syndrome: diagnosis and management. Am Fam Physician. 2012;85(4):352-358.

22. Brun-Buisson C, Minelli C, Bertolini G, et al. Epidemiology and outcome of acute lung injury in European intensive care units. Results from the ALIVE study. Intensive Care Med. 2004;30(1):51-61.

23. Ferguson ND, Frutos-Vivar F, Esteban A, et al. Clinical risk conditions for acute lung injury in the intensive care unit and hospital ward: a prospective observational study. Critical Care. 2007;11(5):R96.

24. Gajic O, Dara SI, Mendez JL, et al. Ventilator-associated lung injury in patients without acute lung injury at the onset of mechanical ventilation. Crit Care Med. 2004;32(9):1817-1824.

25. Shari G, Kojicic M, Li G, et al. Timing of the Onset of Acute Respiratory Distress Syndrome: A Population-Based Study. Respir Care. 2011;56(5):576-582.

26. Gajic O, Dabbagh O, Park PK, et al. Early identification of patients at risk of acute lung injury: evaluation of lung injury prediction score in a multicenter cohort study. Am J Respir Crit Care Med. 2011;183(4):462470 .

27. Jegal $\mathrm{Y}$, Lee $\mathrm{S}$, Lee $\mathrm{KH}$, et al. The clinical efficacy of GOCA scoring system in patients with acute respiratory distress syndrome. $J$ Korean Med Sci. 2008;23(3):383-389.

28. Ferguson ND, Frutos-Vivar F, Esteban A, et al. Clinical risk conditions for acute lung injury in the intensive care unit and hospital ward: a prospective observational study. Critical Care. 2007;11(5):R96.

29. Trillo-Alvarez C, Cartin-Ceba R, Kor DJ, et al. Acute lung injury prediction score: derivation and validation in a population based sample. Eur Respir J. 2011;37(3):604-609.

30. Ward PA, Hunninghake GW. Lung inflammation and fibrosis. Am J Respir Crit Care Med. 1998;157(4 Pt 2): S123-S129.

31. Matthay MA, Zimmerman GA. Acute lung injury and the acute respiratory distress syndrome: four decades of inquiry into pathogenesis and rational management. Am J Respir Cell Mol Biol. 2005;33(4):319327. 
32. Matthay MA, Folkesson HG, Clerici C. Lung epithelial fluid transport and the resolution of pulmonary edema. Physiol Rev. 2002;82(3):569600 .
33. Ranieri VM, Rubenfeld GD, Thompson BT, et al. The ARDS Definition Task Force. Acute respiratory distress syndrome. JAMA. 2012;307(23):2526-2533. 